Article

\title{
Online Power Management with Embedded Offline-Optimized Parameters for a Three-Source Hybrid Powertrain with an Experimental Emulation Application
}

\author{
Bedatri Moulik * and Dirk Söffker \\ Chair of Dynamics and Control, University of Duisburg-Essen, Lotharstr. 1, Duisburg 47057, Germany; \\ soeffker@uni-due.de \\ * Correspondence: bedatri.moulik@uni-due.de; Tel.: +49-203-379-3422; Fax: +49-203-379-3027 \\ Academic Editor: K. T. Chau \\ Received: 29 February 2016; Accepted: 27 May 2016; Published: 7 June 2016
}

\begin{abstract}
Real-time power management in the presence of one or more reversible energy storage systems is a current issue with hybrid electric vehicles (HEVs). To evaluate the potentials of rule-based power management, optimization with respect to two conflicting objectives, fuel consumption and state of charge (SoC) deviation, is considered in this contribution. A modular structure of power management with decoupled offline and online parts is presented. The online part incorporates look-up tables (LUTs) with parameters from the offline optimization part. This permits an inclusion of more LUTs corresponding to different drive patterns. The goal of this contribution is to combine the real-time applicability of rule-based power management and the multi-objective optimization property of genetic algorithms in a single control strategy. Component aging problems are addressed by suitable design. The influence of sizing is investigated. Finally, an experimental setup consisting of components capable of realizing the dynamics of real powertrain components is realized and introduced. A verification/plausibility assessment of modeled dynamics based on the literature is considered. This newly-introduced concept represents a class of power management, which is easy to implement, can tackle different objectives in real time, and adapt itself to unknown driver demands.
\end{abstract}

Keywords: rule-based power management; optimization; HEV

\section{Introduction}

Due to the growing concerns of the fuel crisis and increasing environmental degradation, hybrid vehicles are being intensively researched. All-electric powertrains with a fuel cell-battery-supercapacitor combination have been considered in [1-5]. In the presence of multiple sources, the distribution of power while ensuring various objectives, such as fuel efficiency, battery aging, etc., can be considered as a vital aspect in hybrid vehicles. Multiple sources can imply both primary and secondary sources or storage elements. The design and application of different power management optimizations are available in the literature. Of these methods, the extent of rule-based methods and multi-objective optimization is given in [6] and briefly summarized in this contribution. This contribution contains parts of the text from [6,7].

Rule-based strategies are generally designed based on heuristics, human expertise or mathematical models and do not require prior knowledge of the drive cycle [8]. These strategies are less complex than other types and can be implemented online and in real time. Their major drawback is that they are not optimal with respect to desired vehicle performance, such as fuel consumption minimization, etc. Several alternatives are available as detailed in [9]. Considering the advantages of rule-based methods and optimal solutions offered by the alternatives, a combination 
of rule-based power management to optimization-based methods can be considered: in [10], dynamic programming (DP) is used to understand the deficiency of rules; in [11], parameter optimization using genetic algorithms (GA) is considered to determine the optimal control variables for fixed parameters; using this as the baseline, equivalent consumption management strategy (ECMS) and route-based strategies are developed; in [12], both GA and DP are used; in [13], ECMS and DP are used, whereas in [14], a combination of rule-based power management with the sophisticated Non-Dominated Sorting Genetic Algorithm II (NSGA II) is considered. The use of multi-objective genetic algorithms, such as NSGA II, is advantageous due to the conflicting nature of optimization goals often encountered in HEVs. In [14], for instance, the two conflicting objectives of fuel consumption minimization and sizing are considered, whereas in [15], the minimization of fuel consumption, as well as battery aging is an issue. Multi-objective algorithms are generally offline implementable, and hence, their integration with online rule-based power management requires decoupling of the offline and online parts. A simple technique described in [9] as a time-invariant feedback controller consists of storing control algorithms in look-up tables (LUTs). The control variable/variables are functions of current driving conditions (like power demand, velocity) and state variables (like SoCs of storage elements). Thus, the advantages of rule-based strategies, namely relatively simpler structure and low computational effort required, can be utilized along with the multi-objective optimization property of offline algorithms. They can also be combined with prediction and real-time control strategies to provide solutions where no pre-defined drive cycle is given. From the concept of embedded-online optimization by using offline algorithms [16], it can be concluded that the embedding of optimization results from a decoupled offline process to an online power management controller is possible. Embedding of offline calculated parameters in the context of rule-based power management has been discussed in $[17,18]$.

Under real driving conditions for which no pre-defined drive cycle can be used, an adaption of the power management strategy is important. Adaptive power management strategies without using car navigation data are discussed in [19]. Two options are mentioned: driving condition recognition based on the history of motion and driving condition prediction based on the history of motion. For both options, the procedure is to prepare optimal databases offline and to match the present ones to previously optimized past ones. The difference between the two options is that, in the first option, optimization of the entire drive pattern is considered, whereas in the second option, segment-wise optimization is carried out. Recognition of the present based on the past is considered in the first option, whereas the present is matched to the past in the second option. Moreover, in the first option, updating of rules to integrate new driving patterns is required at regular intervals. In [20], an adaptive power management based on driving pattern recognition is presented. Here, two separate offline processes are considered. In one process, given drive cycles are used to generate driving patterns. These patterns are classified according to power, such as low, medium and high power demand, and the resulting patterns are stored in look-up tables (LUTs) for online implementation. In the other process, the same drive cycles are analyzed for determining the optimal parameters that minimize fuel consumption. Next, control rules are formulated for a sub-optimal rule-based controller. This controller can be implemented online. In the online process, the classification of the driver velocity into patterns is carried out based on the LUT values from one offline process. The corresponding control rule is determined based on the data from the other offline process, which is optimized before.

Thus, rule-based strategies are online-implementable, real-time control schemes applicable for hybrid electric vehicles. A combination with global optimization methods, such as dynamic programming, multi-objective genetic algorithms, etc., to optimize various objectives is possible. Individually optimizing the power management rules offline, such that the results from the optimization are stored in look-up tables, can be used to tune controller parameters online. The decoupling of the optimization process ensures the use of offline-applicable multi-objective optimization techniques in online power management. Moreover, decoupling leads to a possibility of 
adapting the rule-based controller to real driving scenarios. This can be considered by integrating more LUTs for different driving patterns. Thus, by evaluating the potentials and application prospects of rule-based power management, the foundations for the development of an appropriate optimal power management controller that takes into account multiple optimization objectives can be laid. From the detailed literature review presented in [6] and briefly summarized in this contribution, it can be noted that one generalized power management strategy is required that can deal with multiple objectives online and in real time. The remaining open issue is to realize real-time optimization of power flows such that desired powertrain operations can be achieved. Minimization of fuel consumption and lifetime management of components are the main objectives to be considered. Moreover, the design of a suitable structure to accommodate varying driving patterns is also a necessity. With respect to the above-mentioned concerns, a suitable concept is developed in this contribution.

The HEV powertrain considered in this work is an all-electric powertrain. The configuration comprises a fuel cell as the primary energy source and a battery-supercapacitor combination as the storage unit. According to [1], the efficiency of all-electric hybrid vehicles depends on the capability of the energy storage unit. The concept of hybrid storage systems (HESS) was previously proposed in [21-23] and later elaborated in [24]. This concept can be used to overcome the problems faced by batteries, that is low charge/discharge efficiency and short cycle life, by utilizing the properties of a supercapacitor. This is possible due to the better power density of supercapacitors and the better energy density of batteries. By combining the two, an improvement in overall performance can be achieved, as the supercapacitor acts as a support to the batteries and is much more robust in handling surge current. A combination of battery-supercapacitor leads to a significant improvement in fuel economy [25]. The advantages of HESS are detailed in [1,2,24]. Based on the advantages of the fuel cell-battery-supercapacitor hybrid powertrain as stated in [1,3] and utilizing the benefits of the battery-supercapacitor combination, a three-source HEV is considered in this contribution. The optimal utilization of each of these sources is a task of power management. The DC/DC converter plays a central role in power management, and it serves two important purposes: to maintain a constant bus voltage and to send desired current requests to the three sources as defined by the power management. In spite of the benefits offered by multiple input DC/DC converters, such as reduced overall size, weight, losses and cost, three single input DC/DC converters are considered in this contribution for the sake of modeling simplicity. This is because the main objective is to develop an appropriate power management concept that determines the optimal distribution of power between the sources.

Mathematical models of hybrid vehicle components are often required for detailed analysis of powertrain performance and power management strategies. Simulation of these models is the first step for realizing their behavior in experimental setups and real driving scenarios. For a given drive cycle, the vehicle energy losses and its performance can be calculated backwards, as mentioned in [9]. For an unknown velocity pattern, forward modeling becomes necessary, as introduced in [26]. It is possible to combine a backward model with a forward model to measure the drivability error or to model an entire vehicle in a forward manner to investigate real-time systems where the drive cycle is not given. Depending on whether the HEV powertrain is forward or backward modeled, the individual components have to be modeled accordingly. In [27], both quasi-static and dynamic models are considered, where the reason for considering a dynamic model of the battery is stated. Therefore, it is possible to consider quasi-static models of the inverter, motor and vehicle. Components like batteries and supercapacitors, where the state-of-charge is an unknown result of power management and optimization algorithms, need to be modeled dynamically. A fuel cell, on the other hand, can be modeled based on experimentally-determined parameters, as detailed in [28]. Thus, instead of a complex dynamical model as developed in [28], a quasi-static model can be used based on look-up table values of experimentally-determined parameters in [28]. A DC/DC converter, which is an essential component in pure-electric powertrains, can be dynamically modeled like the battery and supercapacitor. 
The paper is organized as follows: the topologies with HESS are briefly introduced followed by the configuration chosen for this contribution; next, the modeling and sizing of powertrain components is detailed followed by a verification of their dynamics based on the literature; next, the concept of emulation is described followed by a possibility to realize powertrain dynamics with controllable sources and sinks; in the next section, the developed power management optimization concept is explained in detail. The operation of the different blocks and the interactions between them is described. Finally, the simulation and emulation results are presented followed by a summary and conclusions.

\section{Possible Topologies and Considered Configuration}

The topologies resulting from hybridization of the storage unit are classified as passive, semi-active and active hybrids [2], as shown in Figure 1. This classification is based on the presence/absence/position of DC/DC converters. One of the three topologies, the parallel active topology, is considered as the best option in [2] and has therefore been chosen in this work.

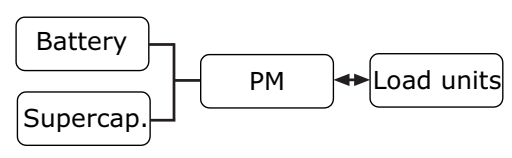

(a)

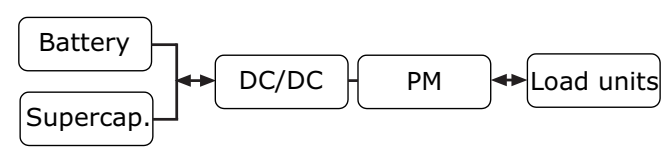

(b)

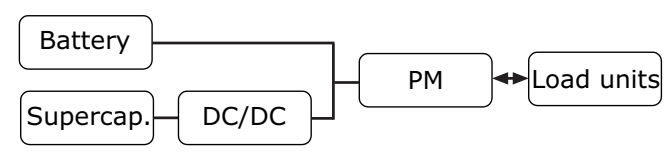

(c)

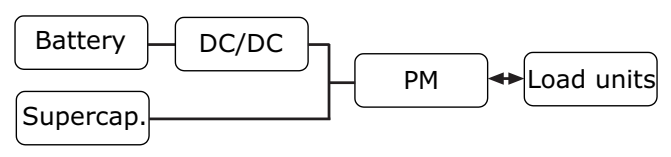

(d)

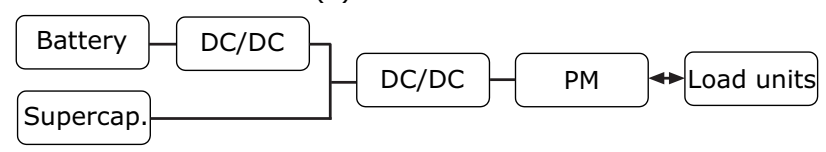

(e)

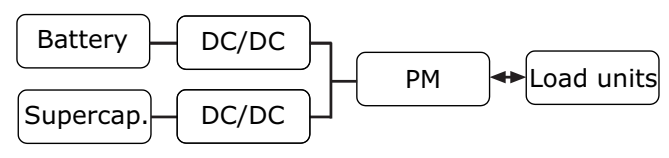

(f)

Figure 1. Typical topologies in electrical powertrains: (a) passive; (b) parallel semi-active; (c) capacitor semi-active; (d) battery semi-active; (e) series active; (f) parallel active (PM refers to power management, supercap. refers to supercapacitor).

The considered HEV powertrain consists of three sources, namely fuel cell, battery and supercapacitor, each accompanied by a DC/DC converter. This part is modeled in a forward manner. The vehicle together with motor and AC/DC converter is modeled in a backward manner. The New European Drive Cycle (NEDC) drive cycle is given as the input. The link between the forward and backward parts is the power management controller. This controller checks the power demand and requests the 
power from sources by sending control signals to DC/DC converters. The considered configuration is shown in Figure 2. The control output from the supervisory controller can be fed to the backward part with the help of a driver model, which can be a simple PI-controller [29] or an imitation of a real human driver [26]. As a first step, the driver model is not considered, but the difference between the power demand from the backward part and the power available from the forward part is compared to analyze the working of the supervisory controller. According to [9], the electrical coupling of the quasi-static backward part and the dynamic forward part by a common bus is possible. The bus power in that case will simply be the sum of all three output power from the three DC/DC converters, such that the required or demanded power is always satisfied.

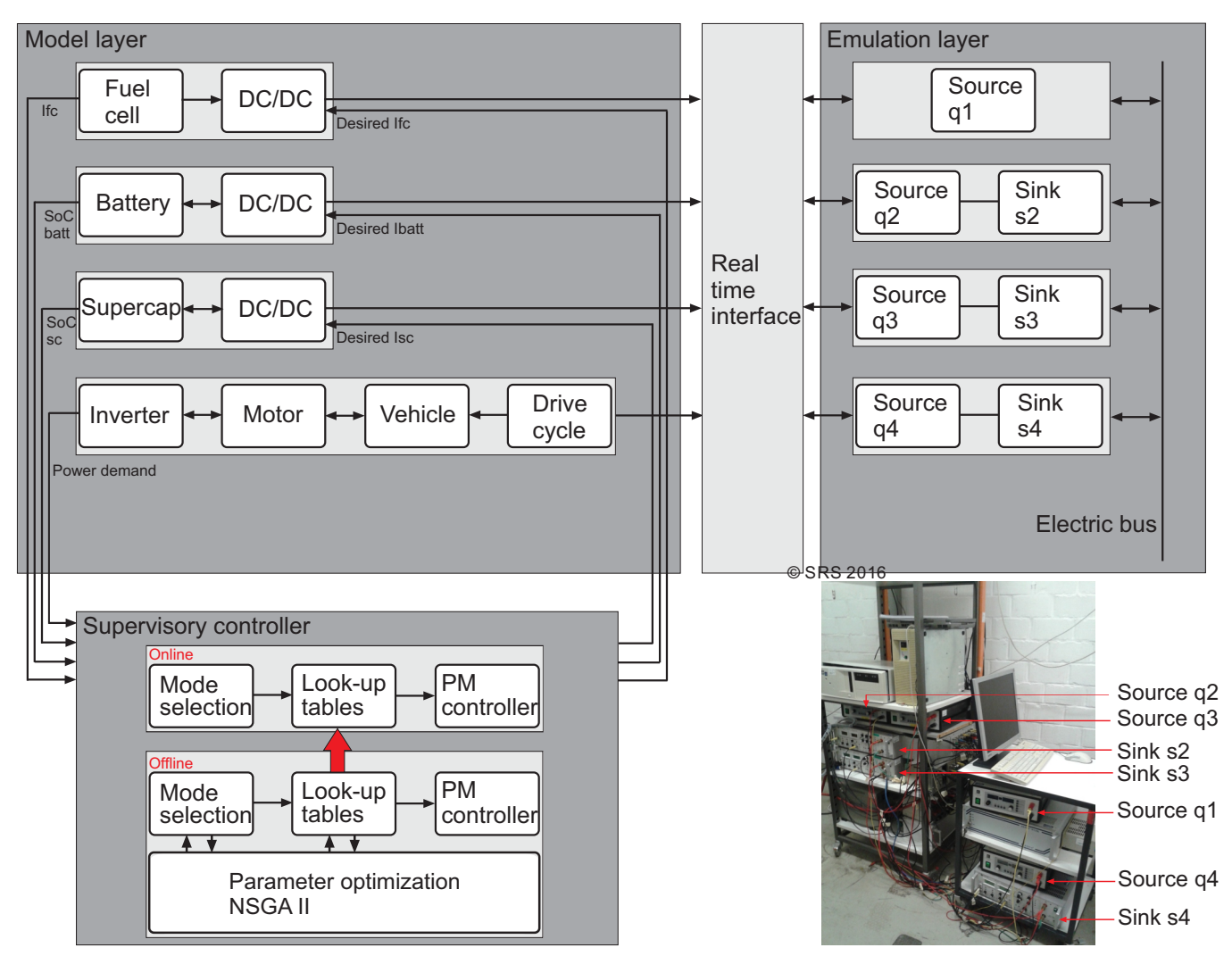

Figure 2. Configuration of an HEV along with the power management optimization. NSGA II, Non-Dominated Sorting Genetic Algorithm II.

\section{Modeling and Sizing of Components}

The drive cycle chosen in this work is the New European Drive Cycle (NEDC). It is fed to a backward modeled powertrain comprised of the vehicular dynamics, an electric motor/generator modeled according to [9,30] and an inverter according to [30]. The forward modeled powertrain is comprised of a fuel cell with look-up tables (LUTs) from a validated model, dynamically-modeled battery, supercapacitor and DC/DC converters. In this contribution, specific modeling approaches are chosen from the literature, where the models have been experimentally validated. Since the focus is on power management optimization, a detailed analysis of different experiment-based modeling approaches is not considered. However, the correctness of these easy-to-realize models is verified by comparing the dynamic behaviors of real components, as reported in the literature. Here, verification is used to state that the results are in accordance with literature, whereas validation is used to define the correctness of those results by related experiments. 


\subsection{Fuel Cell}

Fuel cells are electrochemical devices that convert chemical energy to electrical energy. Unlike internal combustion engines, they deliver pure electrical energy. Fuel cells are more efficient as compared to internal combustion engines [31]. The actual energy conversion efficiency in a fuel cell is about $65 \%$, whereas in an internal combustion engine, it is about $30 \%-35 \%$ [31]. Moreover, by utilizing hydrogen as the energy source, the dependency on fossil fuels can be reduced. The by-products of the electrochemical reaction are generally water and electrical energy, unlike the pollutants released by fossil fuel use.

In a fuel cell, the two electrodes, anode and cathode, are separated by an electrolyte that conducts the free electrons generated as a result of chemical process. The electrons transferred to the cathode side can be used to conduct electricity. The maximum voltage over the anode and cathode are calculated in [28] according to Gibbs free energy:

$$
\Delta G_{f}=G_{f, \text { products }}-G_{f, \text { reactants }}, \Delta G=237.2 \mathrm{KJ} / \text { mole }
$$

The maximum voltage of an individual cell is given by:

$$
v_{0}=\frac{\Delta G_{f}}{N F}, v_{0}=1.2999 \text { volts }
$$

where $N$ denotes the Avogadro number and $F$ denotes the Faraday constant. The typical relation between individual cell voltage $v_{f c}$ and current density $i$ is given in [9] along with the dependence of $v_{f c}$ on irreversible losses, called polarizations. In [28], a real fuel cell from the Ballard Nexa power module is considered, which has a rated output of $1.2 \mathrm{~kW}$ at $26 \mathrm{~V}$ and $46 \mathrm{~A}$ and consumes 18.5 SLPM (standard L/min) of hydrogen. The output voltage varies between 22 and $50 \mathrm{~V}$. The fuel cell system considered is firstly mentioned in [28]; modeling details and fundamentals are given.

Based on the results obtained in [28], a fuel cell model based on look-up tables (LUTs) has been considered in this work. The used LUTs represent the fuel cell voltage-current, and efficiency-power relations are shown in Figures 3 and 4, respectively.

To determine the size of the fuel cell in the considered configuration, the total energy demand corresponding to the given drive cycle is calculated as:

$$
E_{\text {total }}=3821 \mathrm{~kJ}
$$

resulting in an average power of $P_{\text {avg }}$, calculated over $t_{\text {total }}=1200 \mathrm{~s}$ as follows:

$$
P_{\text {avg }}=\frac{E_{\text {total }}}{t_{\text {total }}}=3.184 \mathrm{~kW}
$$




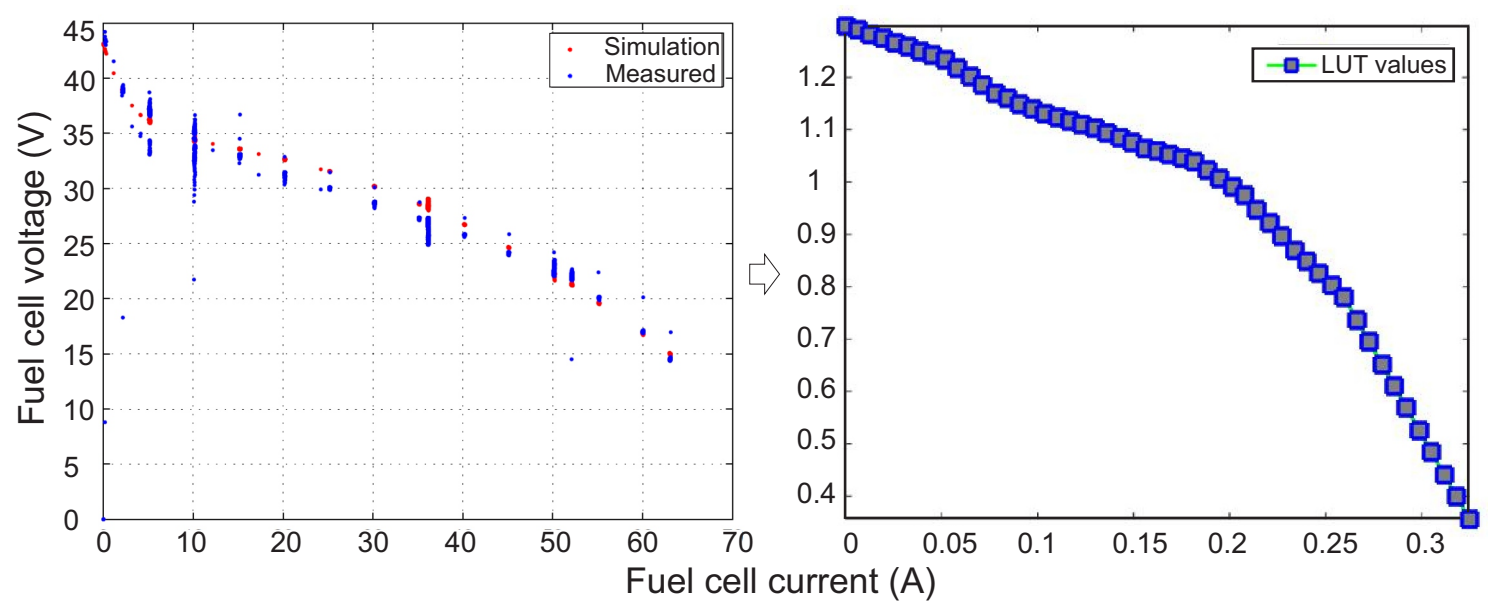

Figure 3. Extrapolation of V-I characteristics.

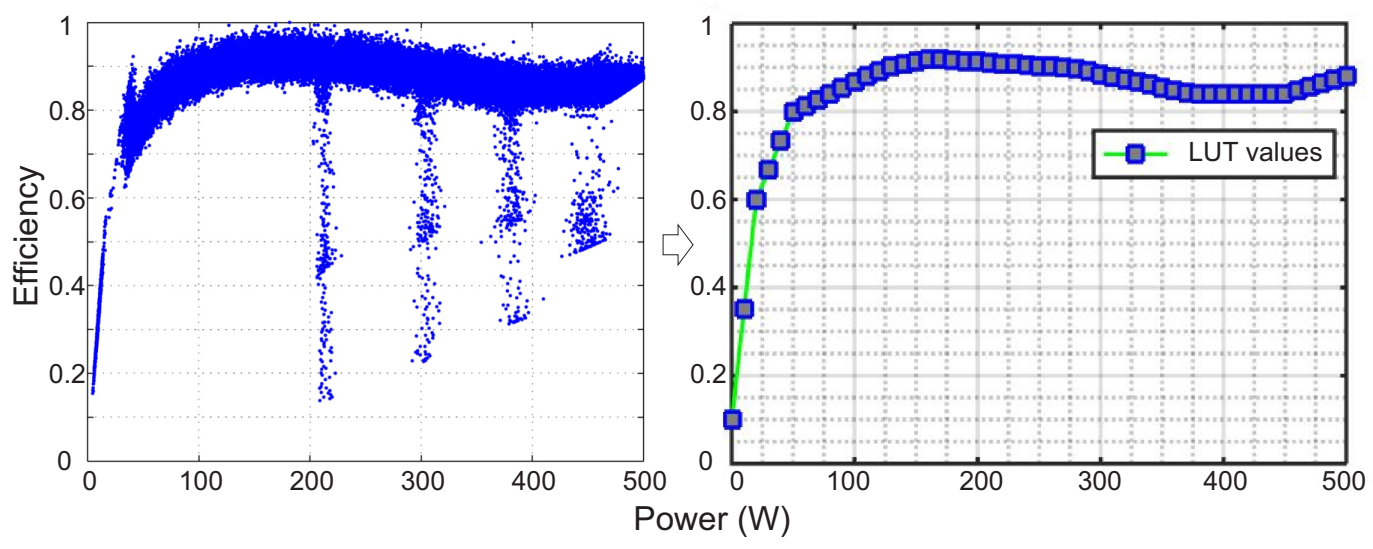

Figure 4. Extrapolation of power-efficiency characteristics.

The fuel cell, as a primary energy source, should be able to supply the total required power as demanded by the given drive cycle. Considering the losses of the DC/DC converter (with $\mu_{D C D C, \text { nor }}=0.98 \%$ approximately), together with the losses of the fuel cell (with an optimum efficiency of $\mu_{F C, o p t}=0.92 \%$ ), the average fuel cell power $P_{F C, a v g}$ can be calculated as:

$$
P_{F C, a v g}=P_{a v g} \cdot \frac{1}{\mu_{D C D C, n o r}} \cdot \frac{1}{\mu_{F C, \text { opt }}}=3.515 \mathrm{~kW}
$$

The power management strategy must consider both operating the fuel cell in its most efficient region, as well as the peak loads of the power demand. The sizing of the fuel cell can done by changing the factor $n_{f c}$.

The long time constant limits the performance of fuel cells in HEVs [1]. According to [1], the efficiency and range of HEVs depend on the capability of the energy storage unit. A possible option is to combine a fuel cell with a battery, but due to the disadvantages posed by batteries, supercapacitors can be used as an option [3]. According to [3], the simultaneous use of batteries and supercapacitors can lead to a promising solution. Therefore, both batteries and supercapacitors are considered in this work in addition to fuel cells to overcome the disadvantages of fuel cells (time constants, aging, etc.). 


\subsection{Battery}

Batteries are electrochemical storage components where energy that is chemically bound is converted to electrical energy, just like the fuel cells; in batteries, the process is reversible. Chemical energy is converted to electrical energy and vice versa. According to [9], a battery can be modeled as a large capacitor. The chemical processes in the electrolyte can be represented by an internal resistance. According to [32], to ensure safe and reliable battery operation, battery management (or power management) systems are important. For this purpose, monitoring of temperature, voltages and currents is required, and an estimation of states, like SoC, is needed. Commonly-used models are electrochemical models and equivalent circuit models. A comparison and evaluation of seven battery models have been presented in [32]. As electrochemical models are complex and deal with a large number of unknown parameters, only equivalent circuit models are considered in this contribution. As detailed in [32], some of the equivalent circuit models are the Thevenin, Rint and DP models. The disadvantage is that the relation between internal resistance and current is not considered. In reality, internal resistance is related to non-linear processes for which electrochemical models are required [9]. In [33], the non-linearity of battery resistance is investigated, and the dependency of impedance on factors, such as SoC, is studied. According to [9], an alternative solution is to develop black box models using experimental data derived from constant current discharge tests. Fitting techniques can then be used to obtain input-output relations. Developing accurate methods to estimate states, like SoC, is a challenge. A comparison of different approaches to estimate battery states is given in [32]. The approach called 'Coulomb counting' is briefly described in [9] along with quasi-static and dynamic modeling of batteries. The quasi-static model is based on the Rint model and the dynamic model based on the Thevenin model.

In this work, a Li-ion battery is chosen, and the battery is modeled using a dynamic modeling approach according to [34,35]. With the help of dynamic models, the transient behavior of the battery can be described [9]. Inductive and capacitive effects are taken into account, and a model-based determination of $\mathrm{SoC}$ is possible [9]. The circuit diagram is shown in Figure 5. In one circuit, the overall capacity of the battery is represented, while in the other, the internal resistance and other dynamic effects. The SoC of the battery is determined from the total capacity $C_{c a p}$ and the battery current $I_{b a t}$.

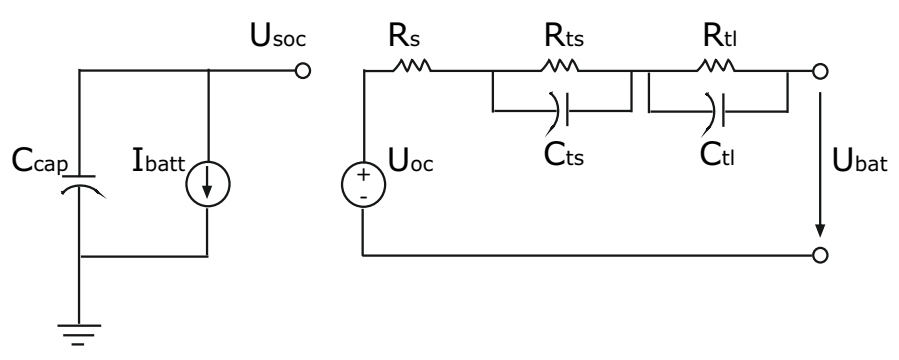

Figure 5. Circuit diagram of the battery model.

The voltage source linking the two circuits, represents the non-linear relation between the battery state of charge and open circuit voltage $U_{o c}$. This non-linear relation between SoC and $U_{o c}$ using 33 cells can be represented by a LUT, as shown in Figure 6. 


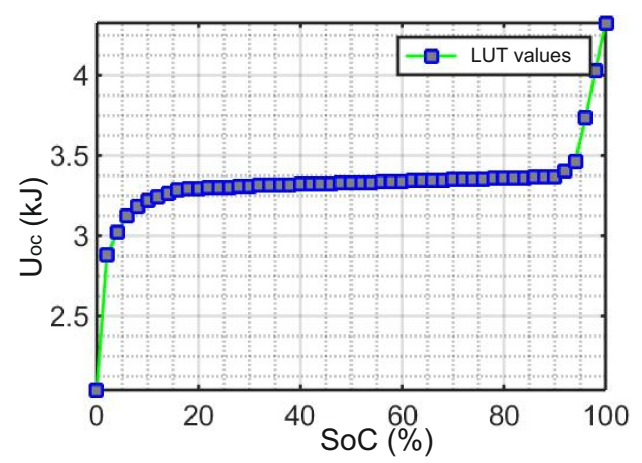

Figure 6. Relation between SoC and voltage.

According to $[34,35]$, by solving the equation for the circuit, state space models can be generated:

$$
\dot{\mathbf{x}}=\left[\begin{array}{ccc}
0 & 0 & 0 \\
0 & -\left(R_{t s} C_{t s}\right)^{-1} & 0 \\
0 & 0 & -\left(R_{t l} C_{t l}\right)^{-1}
\end{array}\right] \mathbf{x}+\left[\begin{array}{l}
-C_{c a p}^{-1} \\
-C_{t s}^{-1} \\
-C_{t l}^{-1}
\end{array}\right] \mathbf{u}
$$

with,

$$
\begin{gathered}
\mathbf{x}=\left[\begin{array}{c}
U_{s o c} \\
U_{t s} \\
U_{t l}
\end{array}\right], \mathbf{u}=I_{b a t} \\
y=U_{b a t}=g\left(U_{s o c}\right)+U_{t s}+U_{t l}-R_{s} \cdot I_{b a t}
\end{gathered}
$$

where battery current $I_{b a t}$ is the input and terminal voltage $U_{b a t}$ is the output.

In [34], first an initial model is built with parameters based on the literature. Then, the $U_{o c}$-SoC relationship is derived from experiments on a real battery; then a refined model is built based on the $U_{o c}-$ SoC relationship; and finally, the resistances and capacitances, namely $R_{t s}, C_{t s}, R_{t l}, C_{t l}$ and $R_{S}$, are estimated. The experimental setup with the real battery connected to programmable load is given in [34]. After a constant resistance discharge test, the SoC over the entire test is calculated by integrating the current as:

$$
U_{s o c}=-\frac{1}{C_{c a p} \cdot \int I_{b a t}} \Rightarrow \dot{U}_{s o c}=-\frac{I_{b a t}}{C_{c a p}}
$$

where normalized values of $U_{s o c}$ from 0-1 volts correspond to SoC values between $0 \%$ and $100 \%$.

The relationship between $U_{o c}$ and $U_{s o c}$ or SoC is given by:

$$
U_{o c}=g\left(U_{s o c}\right)
$$

and can be established with the help of a LUT [36], as shown in Figure 6.

The specifications of the real Li-ion battery (used in [34]) as available from the manufacturers is: maximum cell voltage as $4.2 \mathrm{~V}$ and capacity as $60 \mathrm{Ah}$. For a fully-charged battery, $U_{s o c}$ should be $1 \mathrm{~V}$, but as $4.09 \mathrm{~V}$ is measured in [34] instead of the specified value by the manufacturer, that is 4.2 , so instead of $1 \mathrm{~V}, 0.9 \mathrm{~V}$ is calibrated for full charge.

Estimation of the values of $R_{t s}, C_{t s}, R_{t l}, C_{t l}$ and $R_{s}$ are given in [34], where constant current discharge tests are carried out. The load is switched between $3.6 \mathrm{~A}$ and $0 \mathrm{~A}$ by the programmable sink. The battery is discharged over nine cycles (constant current followed by rest), the beginning of each cycle depending on when a specific $U_{s o c}$ value is reached. The test data are analyzed for the discharge and rest phases leading to the estimation of the resistances and capacitances. In [34], this is done using the curve fitting toolbox. The parameters are plotted as a function of SoC. These variations of resistances and capacitances with SoC can either be implemented as LUTs or assumed 
as constants (averaged values over nine discharge cycles). The numbers are assumed as constants to avoid unnecessary model complexity in this contribution.

For the determination of the battery size, the ability of the battery to supply the maximum peak of power can be considered. The maximum energy to be stored is given for the corresponding time period of the assumed drive cycle as:

$$
E\left(t_{0}=820 s\right)-E\left(t_{1}=1144 s\right)=3.1 \mathrm{MJ}
$$

with a factor $k=2$, which takes into account the losses while the battery is discharging and charging.

The maximum energy to be stored can be considered as:

$$
E_{b a t}=6.2 \mathrm{MJ}
$$

The model design parameters and capacity of the battery can be adjusted according to this value. In spite of the design considered, the actual maximum power delivered by the battery will be defined by the power management strategy. The power management will keep the battery operation within the limits by using the second storage element, namely the supercapacitor. It should be noted that the battery model is not designed based on the charging/discharging limits of a real battery. A real battery is equipped with a battery management system that keeps the SoC within operation limits $(15 \%-95 \%)$. In this work, this feature is considered to be a task of power management.

\subsection{Supercapacitor}

Supercapacitors (also known as ultracapacitors or double-layer capacitors) are capable of storing a large amount of energy as compared to conventional capacitors. Their specific power is much higher than batteries, but the specific energy is lower. Due to their higher power density, they can be used in hybrid powertrains to realize fast transient power demands. Here, the energy is stored as a result of charge separation. The charge separation occurs between the layers that separate the electrolyte and the electrodes. According to [37], the difference in the way energy is stored in the battery and in the supercapacitor is that, in the battery, an indirect storage via an electrochemical process is used. In a supercapacitor, a direct storage of charge as a result of electrostatic process occurs. The difference in the discharge curves of the battery and supercapacitor is given in [37].

The supercapacitor model considered in this work is based on the models used in $[37,38]$. As shown in Figure 7, the main capacitance $C_{S C}$ is connected in parallel to a resistance $R_{2}$, which represents the self-discharge of supercapacitors. The RCnetwork is connected to another RC network consisting of capacitance $C_{p}$ and resistance $R_{p}$. The charge/discharge losses are represented by the resistance $R_{1}$, and the resistance used for protecting the supercapacitor against overcharge is represented by $R_{3}$. Considering that the switch is open,

$$
I_{S C}=I_{\text {norm }}
$$

and,

$$
U_{S C}=U_{1}+U_{p}+U_{2}
$$

where,

$$
U_{1}=R_{1} \cdot I_{\text {norm }}
$$




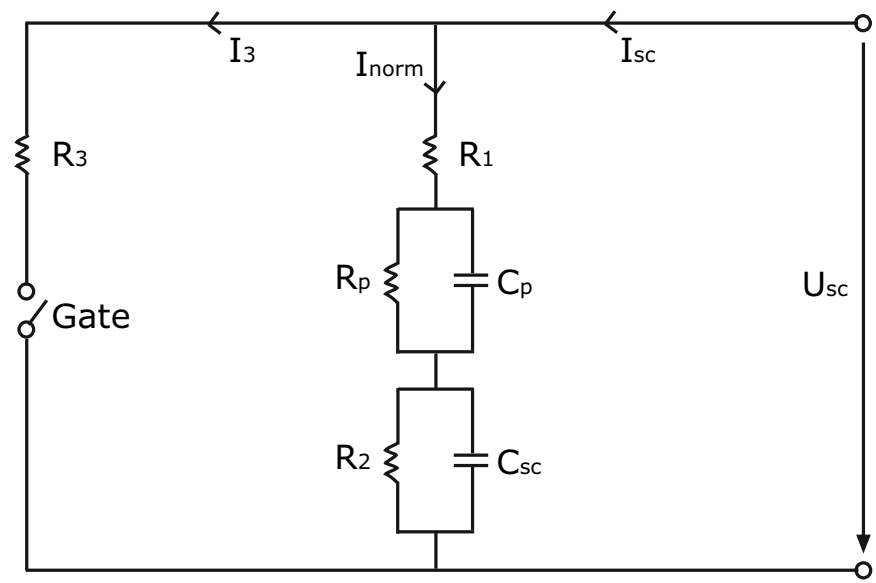

Figure 7. Circuit diagram of the supercapacitor model.

For the RC network, the following equations can be derived:

$$
\begin{gathered}
\dot{U}_{p}=\frac{I_{\text {norm }}}{C_{p}}-\frac{U_{p}}{R_{p} \cdot C_{p}} \\
\dot{U}_{2}=\frac{I_{\text {norm }}}{C_{S C}}-\frac{U_{2}}{R_{2} \cdot C_{S C}}
\end{gathered}
$$

From Equations (14)-(17), the following state space equations:

$$
\dot{\mathbf{x}}=\left[\begin{array}{cc}
-\left(R_{p} C_{p}\right)^{-1} & 0 \\
0 & -\left(R_{2} C_{S C}\right)^{-1}
\end{array}\right] \mathbf{x}+\left[\begin{array}{c}
C_{p}^{-1} \\
C_{S C}^{-1}
\end{array}\right] \mathbf{u}
$$

where,

$$
\begin{gathered}
\mathbf{x}=\left[\begin{array}{l}
U_{p} \\
U_{2}
\end{array}\right], \mathbf{u}=I_{\text {norm }} \\
y=U_{S C}=R_{1} \cdot I_{\text {norm }}+U_{p}+U_{2}
\end{gathered}
$$

can be derived.

The extraction of model parameters is done based on a real supercapacitor model [37]. The data available from manufacturers are: nominal voltage: $14 \mathrm{~V}$, nominal capacity: $350 \mathrm{~F}$; mass: $24 \mathrm{~kg}$. In [37], constant current tests are carried out with the real supercapacitor by connecting it to a programmable load. The supercapacitor is charged at $10 \mathrm{~A}$ until the voltage reaches $11 \mathrm{~V}$, then the current is cut off for about $10 \mathrm{~min}$, and a constant current discharge phase follows at $-20 \mathrm{~A}$. For the calculation of $C_{S C}$, the time period when the voltage rise from $2 \mathrm{~V}-9 \mathrm{~V}$ is chosen, namely $\Delta t=225 \mathrm{~s}-46 \mathrm{~s}=209 \mathrm{~s}$. Therefore,

$$
C_{S C}=\frac{\Delta Q}{\Delta U}
$$

and:

$$
Q=\int i(t) d t
$$

where, $\Delta t=209 \mathrm{~s}$ and $\Delta U=9 \mathrm{~V}-2 \mathrm{~V}=7 \mathrm{~V}$,

$$
Q=10 \mathrm{~A} * 209 \mathrm{~s}=2090 \mathrm{C}
$$

and,

$$
C_{S C}=\frac{2090}{7} \approx 300 \mathrm{~F}
$$


The value of capacitance $C_{p}$ is chosen as one-thirteenth of $C_{S C}$.

To estimate $R_{1}$, the phase right after the charge current is cut off is taken into account. This corresponds to [37] as:

$$
R_{1}=\frac{\Delta U}{\Delta i} \approx 2 \mathrm{~m} \Omega
$$

The resistance $R_{2}$ is estimated as $200 \Omega$. To estimate $R_{3}$, the voltage drop after turning off of the charging current is considered as:

$$
R_{3}=\frac{\Delta t}{-\ln \left(\frac{U_{1}}{U_{0}}\right) \cdot C_{S C}} R_{3}=10 \Omega
$$

The parameters chosen to model the supercapacitor in this work are taken from [37]. The sizing of the supercapacitor is determined by arranging the capacitors in series and parallel. The nominal voltage of the supercapacitor $V_{n}=14 \mathrm{~V}$ is not enough to maintain a constant bus voltage of $500 \mathrm{~V}$. It can be achieved by a series arrangement of supercapacitors as follows:

$$
U_{\text {total }}=\sum_{n=0}^{\text {seriessC }} U_{S C, n}
$$

where series $S_{S C}$ is the number of capacitors arranged in series. Thus, the total capacitance is reduced to:

$$
\frac{1}{C_{\text {total }}}=\sum_{n=0}^{\text {seriessC }} \frac{1}{U_{S C, n}}
$$

If series $_{S C}$ is chosen as 36 , then the maximum voltage $V_{n, t o t a l}$ will be:

$$
V_{n, \text { total }}=V_{n} \cdot \text { series }_{S C}=504 \mathrm{~V}
$$

Similarly, the parallel arrangement of capacitors causes an increase in the total capacitance as:

$$
C_{\text {total }}=\sum_{n=0}^{\text {parallel }_{S C}} C_{S C, n}
$$

where parallel $_{S C}$ is the number of capacitors arranged in parallel. Thus, the storage capacity of the supercapacitor can be adjusted by changing the configuration. Deciding on the battery dimensions, here a safety factor of $k=2$ is considered, so the maximum energy to be stored is given by:

$$
E_{S C}=6.2 \mathrm{MJ}
$$

To achieve this, a parallel configuration of supercapacitors such that parallel $_{S C}=4$ is necessary. Once again, this is to be taken into consideration by the power management strategy.

\subsection{DC/DC Converter}

The purpose of the DC/DC converter in this work is to maintain a constant output voltage despite varying input voltage. It is possible to control the output voltage in order to track a reference, but this makes the DC/DC converter more complex and expensive. The converter should be dynamic enough to respond to the changes in demanded power and make the required power available on the bus. Buck, boost and buck-boost DC/DC converters are the commonly-known types. DC/DC converters can be mono- or bi-directional. Bi-directional DC/DC converters can transfer power to and from the source, thus allowing the regenerated energy to be saved. In this work, two storage elements are considered: battery and supercapacitor. The DC/DC converter model chosen is a CUK-DC/DC converter with buck-boost behavior, in accordance with the model already described in [28]. The 
circuit diagram of the DC/DC converter is shown in Figure 8. The two states of the DC/DC converter can be given as:

1. Gate 0 is closed and Gate 1 open $\Rightarrow u_{0}=0 ; u_{1}=1$,

2. Gate 0 is open and Gate 1 closed $\Rightarrow u_{0}=1 ; u_{1}=2$.

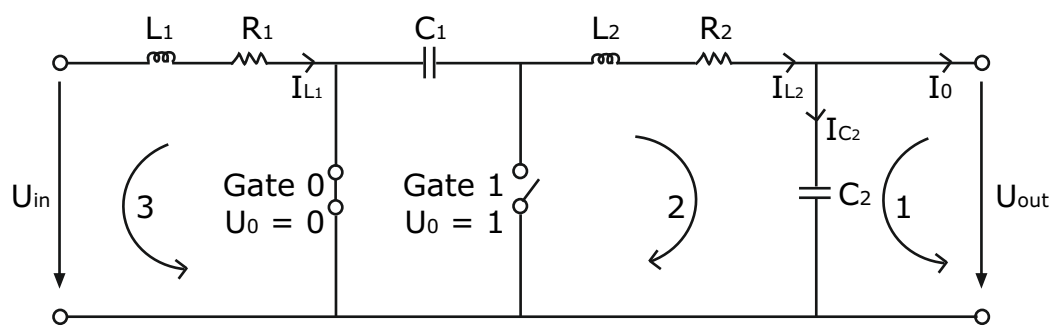

Figure 8. Circuit diagram of the DC/DC converter.

The duty cycle is defined as:

$$
D=\frac{t_{o n}}{t_{o f f}}
$$

From the two states, two equations can be derived as follows:

$$
\dot{U}_{C 1}=\frac{I_{C_{1}}}{C_{1}} ; \dot{U}_{C 2}=\frac{I_{C_{2}}}{C_{2}}
$$

and:

$$
U_{L 1}=\dot{I}_{L 1} \cdot L_{1} ; U_{L 2}=\dot{I}_{L_{2}} \cdot L_{2}
$$

For the first state where $u_{0}=0 ; u_{1}=1$,

$$
-U_{i n}+U_{L 1}+U_{R 1}=0
$$

and:

$$
\dot{I}_{L 1}=-\frac{R_{1} I_{L 1}}{L_{1}}+\frac{U_{i n}}{L_{1}}
$$

For the left loop and right Loops 3 and 2,

$$
U_{C 1}+U_{L 2}+U_{R 2}+U_{C 2}=0
$$

and:

$$
\dot{I}_{L 2}=-\frac{U_{C 1}}{L_{2}}-\frac{U_{C 2}}{L_{2}}-\frac{R_{2} I_{L 2}}{L_{2}}
$$

where,

$$
I_{C 1}=I_{C 2}=I_{L 2}
$$

From Equations (33) and (35)-(39), the model in state space form:

$$
\dot{\mathbf{x}}=\underbrace{\left[\begin{array}{cccc}
-\frac{R_{1}}{L_{1}} & 0 & 0 & 0 \\
0 & -\frac{R_{2}}{L_{2}} & -\frac{1}{L_{2}} & -\frac{1}{L_{2}} \\
0 & \frac{1}{C_{1}} & 0 & 0 \\
0 & \frac{1}{C_{2}} & 0 & 0
\end{array}\right]}_{\mathbf{A}_{\mathbf{1}}} \mathbf{x}+\left[\begin{array}{cc}
\frac{1}{L_{1}} & 0 \\
0 & 0 \\
0 & 0 \\
0 & -\frac{1}{C_{2}}
\end{array}\right] \mathbf{u}
$$


with:

$$
\mathbf{x}=\left[\begin{array}{c}
I_{L 1} \\
I_{L 2} \\
U_{C l} \\
U_{C 2}
\end{array}\right], \mathbf{u}=\left[\begin{array}{l}
U_{\text {in }} \\
I_{\text {out }}
\end{array}\right], \mathbf{y}=\mathbf{x}
$$

can be derived. The input current $I_{L 1}$ is the current signal sent from power management to the DC/DC converter, and this is the current required to be drawn from the sources. The capacitor voltage $U_{\mathrm{C} 2}$ is the output voltage, as the bus voltage is to be held constant.

For the second state where $u_{0}=1 ; u_{1}=2$, the state space model:

$$
\dot{\mathbf{x}}=\underbrace{\left[\begin{array}{cccc}
-\frac{R_{1}}{L_{1}} & 0 & -\frac{1}{L_{1}} & 0 \\
0 & -\frac{R_{2}}{L_{2}} & 0 & -\frac{1}{L_{2}} \\
\frac{1}{C_{1}} & 0 & 0 & 0 \\
0 & \frac{1}{C_{2}} & 0 & 0
\end{array}\right]}_{\mathbf{A}_{2}} \mathbf{x}+\left[\begin{array}{cc}
\frac{1}{L_{1}} & 0 \\
0 & 0 \\
0 & 0 \\
0 & -\frac{1}{C_{2}}
\end{array}\right] \mathbf{u}
$$

with:

$$
\mathbf{x}=\left[\begin{array}{c}
I_{L 1} \\
I_{L 2} \\
U_{C l} \\
U_{C 2}
\end{array}\right], \mathbf{u}=\left[\begin{array}{l}
U_{\text {in }} \\
I_{\text {out }}
\end{array}\right], \mathbf{y}=\mathbf{x}
$$

can be derived.

According to [28], the coupling between $\mathbf{A}_{\mathbf{1}}$ and $\mathbf{A}_{\mathbf{2}}$ can be expressed by the duty cycle $D$ as:

$$
\mathbf{A}_{\text {tot }}=\mathbf{A}_{\mathbf{2}}+D \cdot\left(\mathbf{A}_{\mathbf{1}}-\mathbf{A}_{\mathbf{2}}\right)
$$

For the coupled system, the non-linear state-space equations,

$$
\dot{\mathbf{x}}=\underbrace{\left[\begin{array}{cccc}
-\frac{R_{1}}{L_{1}} & 0 & -\frac{1}{L_{1}} & 0 \\
0 & -\frac{R_{2}}{L_{2}} & 0 & -\frac{1}{L_{2}} \\
\frac{1}{C_{1}} & 0 & 0 & 0 \\
0 & \frac{1}{C_{2}} & 0 & 0
\end{array}\right]}_{\mathbf{A}_{\text {lin }}} \mathbf{x}+\underbrace{\left[\begin{array}{cccc}
0 & 0 & \frac{D}{L_{1}} & 0 \\
0 & 0 & -\frac{1}{L_{2}} & 0 \\
-\frac{D}{C_{1}} & \frac{D}{C_{1}} & 0 & 0 \\
0 & 0 & 0 & 0
\end{array}\right]}_{\mathbf{A}_{\text {nonlin }}} \mathbf{x}+\left[\begin{array}{cc}
\frac{1}{L_{1}} & 0 \\
0 & 0 \\
0 & 0 \\
0 & -\frac{1}{C_{2}}
\end{array}\right] \mathbf{u}
$$

with:

$$
\mathbf{x}=\left[\begin{array}{l}
I_{L 1} \\
I_{L 2} \\
U_{C l} \\
U_{C 2}
\end{array}\right], \mathbf{u}=\left[\begin{array}{l}
U_{\text {in }} \\
I_{\text {out }}
\end{array}\right], \mathbf{y}=\mathbf{x}
$$

can be derived.

Thus, the state space form of DC/DC converter model has a linear system matrix $A_{\text {lin }}$ and a non-linear system matrix $A_{\text {nonlin }}$, which is dependent on the duty cycle $D$. According to [39], the DC/DC converter can be internally controlled by a PI-controller with bus voltage as the reference input, and externally, it can be controlled by the power management controller that sends the required current output signal to the converter. The switching losses of the DC/DC converter can be calculated based on a LUT, which is based on validated results from [40]. From this LUT, the energy conversion efficiency $\mu_{D C / D C}$ can be calculated. Using:

$$
P_{\text {out }}=P_{D C / D C} \cdot \mu_{D C / D C}=I_{\text {out }} \cdot U_{C 2} \cdot \mu_{D C / D C}
$$


the output power of the converter $P_{\text {out }}$ can be determined.

\section{Model Verification Based on Literature Results}

To determine the accuracy of the concepts and relations represented by the models, verification is necessary. The importance of laboratory-based tests before real road tests is declared in [41]. A possibility to improve modeling techniques by using data from laboratory tests or experimental papers is also stated. In this contribution, verification is based on a comparison with validated models from the literature.

\subsection{Verification of the Battery Model}

To verify the dynamic behavior of the simulated model, the results are compared to experimentally-validated models of [34-36,42]. The battery is first fully charged then fully discharged in a cyclic manner. A constant current of $I_{b a t}=25 \mathrm{~A}$ is applied till the next SoC level $\left(S_{0} C_{b a t}=10 \%, 20 \%, \ldots\right.$, $90 \%$ ) is reached. At the next SoC level, current is turned off, then turned on again after a 25-min pause. The voltage variation resulting from the SoC- $U_{s o c}$ relationship (Figure 6) is similar to that discussed in the literature. In Figure 9, the voltage variation while the battery is charging is shown. At the moment when the current is turned off, the resulting voltage curve exhibits an initial peak followed by a gradual logarithmic decay. Similarly, when the current is turned on again, a short dip in voltage is followed by a gradual increase. As shown in Figure 9, this behavior is inverted during the discharging process. In Figure 10, the SoC variation is shown. The SoC and energy saved in the battery increase continuously with each charge cycle. In reality, the energy supplied to the battery is not always the same as the energy that the battery is capable of supplying. The supplied energy or the power required to charge the battery depends on the battery voltage. During the charge-discharge cycles, a part of this energy is lost in irreversible chemical processes.
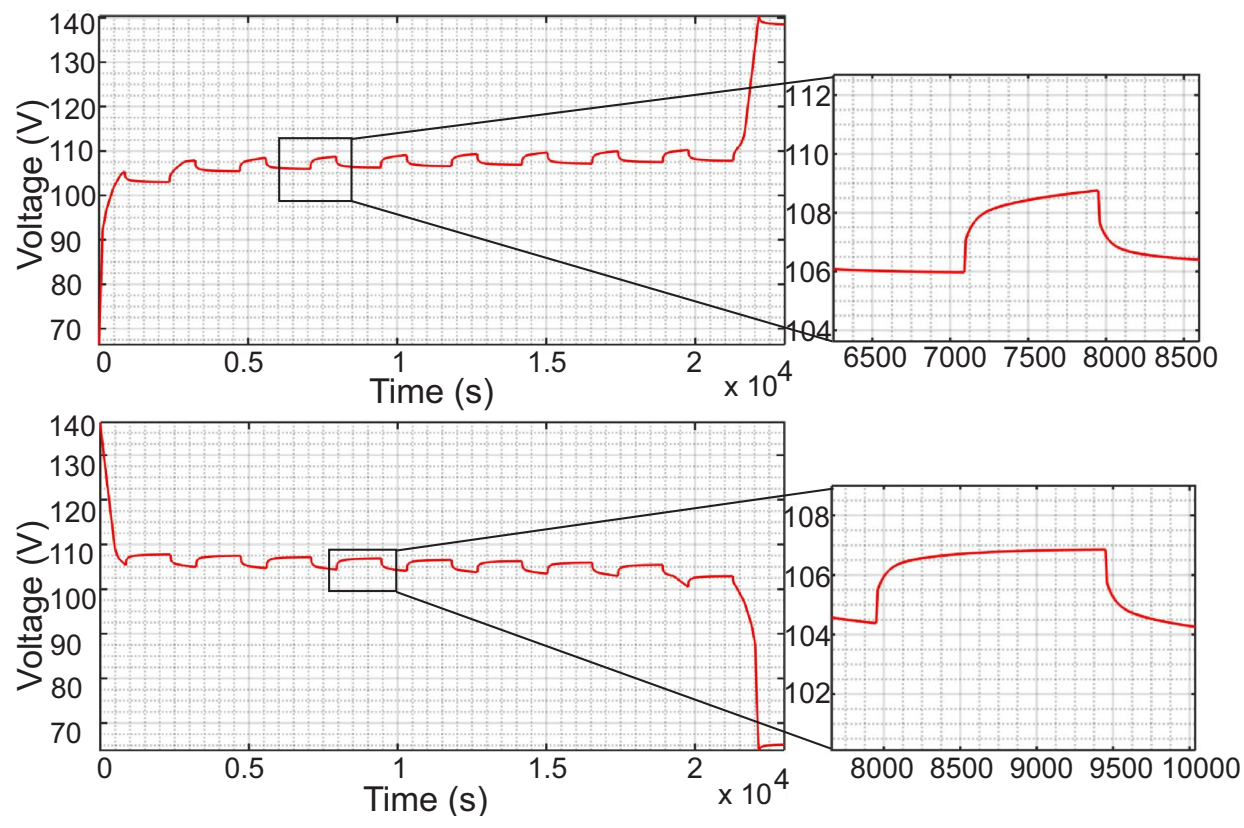

Figure 9. Battery voltage variation during charging and discharging. 

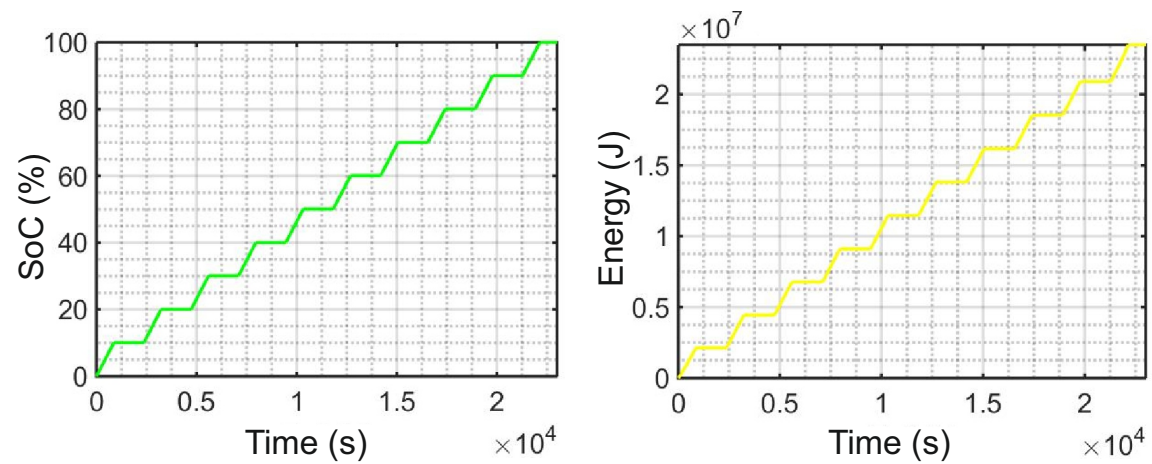

Figure 10. Battery SoC and energy during charging.

\subsection{Verification of the Supercapacitor Model}

To verify the simulation model, tests according to [37] are carried out. First, the supercapacitor is charged with a constant current of $10 \mathrm{~A}$ till $U_{S C}$ is almost equal to $11 \mathrm{~V}$; then, current is turned off, and after an 8-min pause, the supercapacitor is completely discharged with $-20 \mathrm{~A}$. As shown in Figure 11, the voltage of the supercapacitor increases till the current is turned off. At the moment when the current is turned off, a small peak is followed by a gradual decay of voltage. This is due to the self-discharging tendency of supercapacitors. By comparing the response of the supercapacitor while charging and discharging (Figure 11) with the experimentally-determined results of [37,43], the correctness of modeling can be confirmed.

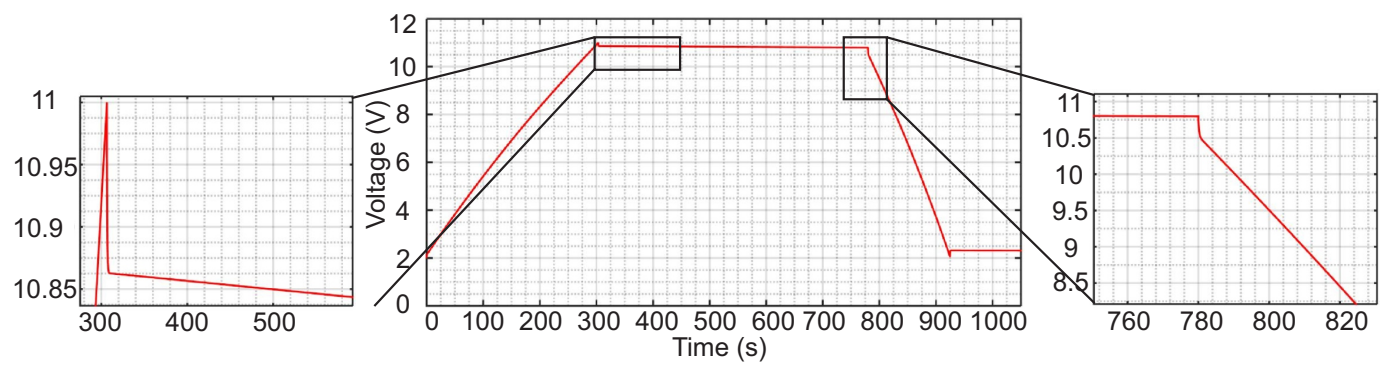

Figure 11. Capacitor voltage during charging and discharging.

\subsection{Verification of the DC/DC Converter Model}

To verify the DC/DC converter model, a current $I_{\text {out }}=25 \mathrm{~A}$ is used. The task of the DC/DC converter here is to maintain constant bus voltage. Thus, the load demand to be fulfilled by the source connected to the converter is $12.5 \mathrm{~kW}$. The source connected to the converter during this test is a battery. After $5 \mathrm{~s}$, the current is turned off, and a 2-s pause follows. Then, $I_{\text {out }}$ is changed to $-25 \mathrm{~A}$, so as to charge the battery with $12.5 \mathrm{~kW}$. The result of this test can be seen in Figure 12. Due to the inverted bust-boost behavior, the voltage is negative. If it is multiplied by negative values of $I_{\text {out }}$, which will be the output from the power management and reference input to the converter, the bus voltage can be made positive. As shown in Figure 12, the relatively short oscillatory behavior at the switching moments die down fast due to the control action of the PI-controller in the DC/DC converter. The response to the load demand is the input current to the DC/DC current from the battery. It shows a damped proportional behavior, as shown in Figure 13. A short oscillation at the beginning of the simulation can be detected, but the CUK-DC/DC converter is capable of keeping the ripples as small as possible [44] to avoid extreme current transients. Thus, from the above-mentioned tests, in combination with the validated results from [40], the dynamics of the bi-directional DC/DC converter is verified. 


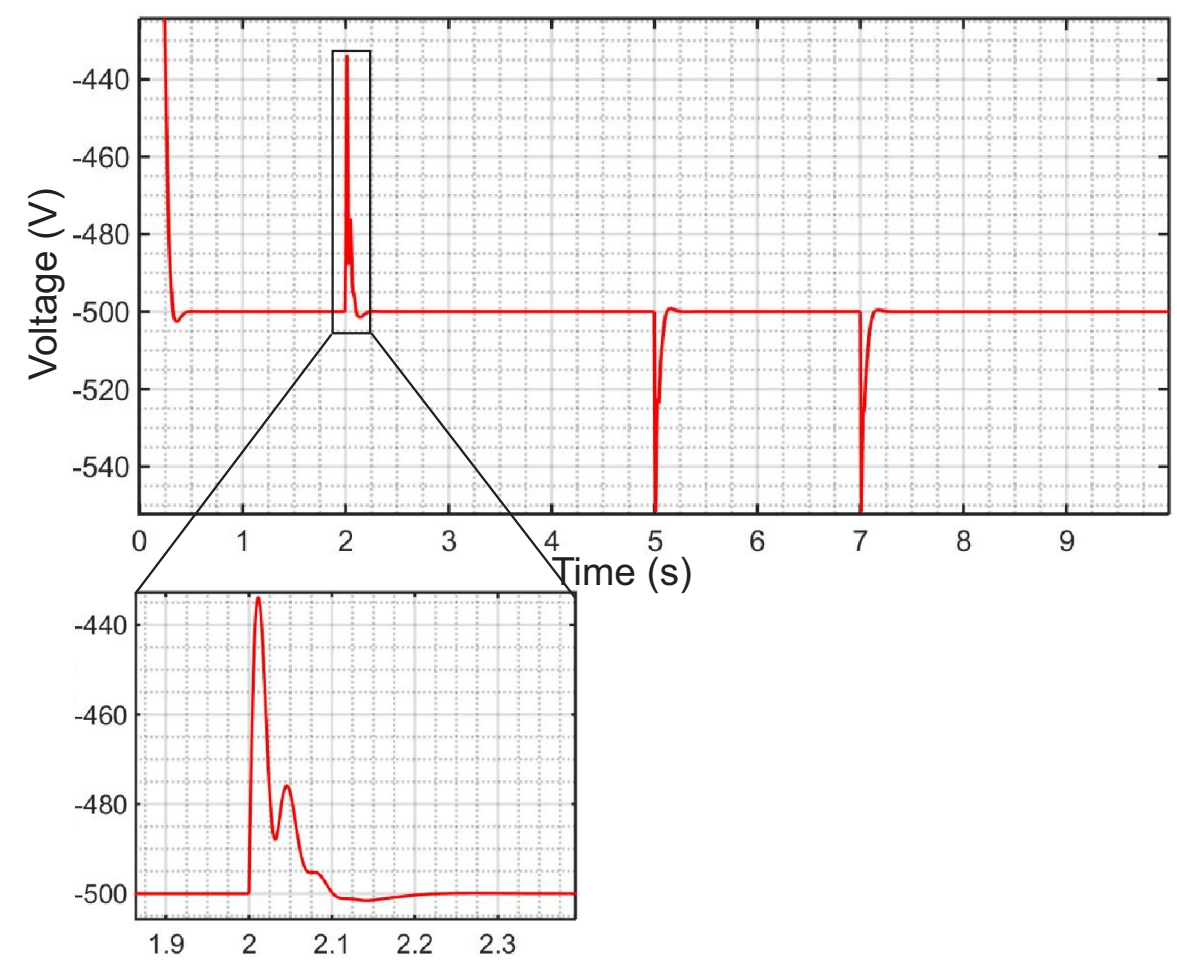

Figure 12. Bus voltage during charging and discharging.

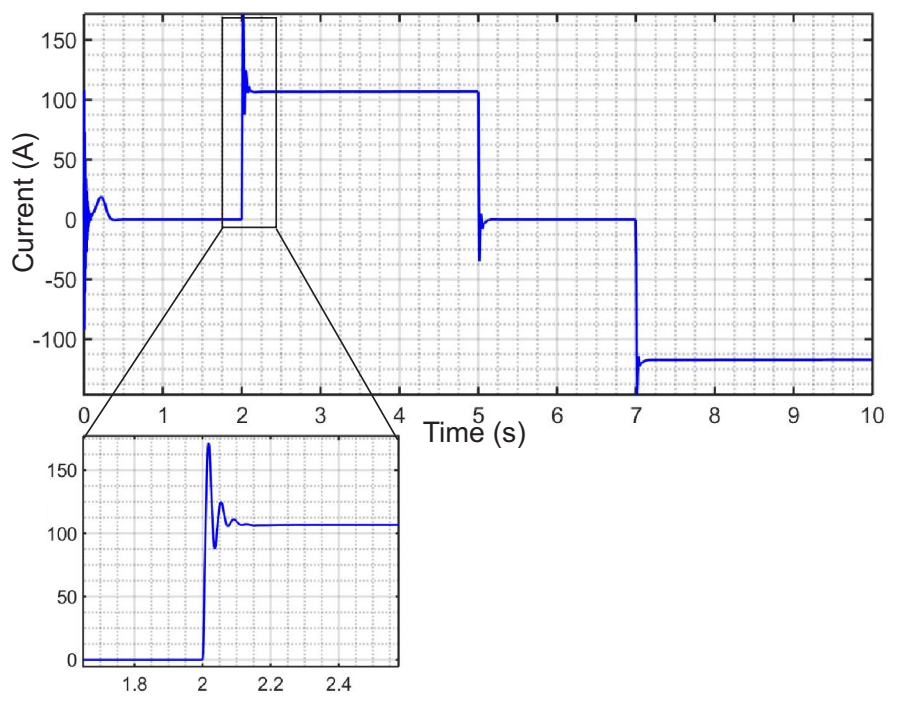

Figure 13. Source current during charging and discharging.

\section{Powertrain Configuration with Emulated Components}

Although modeling and simulation are useful for gaining detailed understanding of system dynamics and behavior, experiments are important for investigating the applicability by the validation of these models. According to [45], validation refers to the precision at which the model represents the physical world, whereas validation experiments are performed to produce data for model validation. For instance, in [41], experiments are performed with the help of a 1:1 scale laboratory-based dynamic setup; in [46], first, experiments are conducted on individual units in stationary conditions to evaluate their behavior at constant electric parameters, then their performance under dynamic operations with real driving cycles is evaluated. Instead of performing experiments with real powertrain 
components, in this contribution, a comparison with experimentally-determined parameters from real components is considered along with a brief introduction of a concept known as emulation. To carry out the power management and control of an analog subsystem integrated with a digital subsystem [47], the emulation of component dynamics has already been discussed in the literature. According to [16], emulation is based on controllable powertrain components, which can be used as a replacement for real components. This solves the problems posed by classical setups, namely high cost, deterioration/damage risks, large energy and fuel consumption, etc.

Emulation of fuel cell dynamics using a programmable power source $[48,49]$ and a power electronic converter [50-52] has been discussed. Similarly, emulation of a supercapacitor [48] and a battery $[47,53]$ using a programmable power source-sink combination has been discussed. In [53], an automotive power net test-bench is mentioned. Here, emulation is based on the fact that physical models can be run on a real-time system and the current calculated from the model set by electronic loads. The test-bench proposed in [53] can be used not only for batteries and supercapacitors, but also systems that behave as controllable source-sink combinations, such as bi-directional DC/DC converters. Load emulation using a dynamically-controllable source-sink [54] to ensure bi-directional power exchange can also be considered. A fuel cell-supercapacitor-based hardware-in-the-loop (HiL) test rig was built [28] at the Chair of Dynamics and Control (University of Duisburg-Essen). It was modified and generalized for the emulation of different powertrains, for example hybrid hydraulic powertrains and wind energy conversion systems [55,56], along with hybrid electric powertrains. In this work, a further generalization is considered by replacing all of the real powertrain components by emulated components. The experimental setup is shown in Figure 2. On the left side is the model layer comprising the powertrain configuration. This simulation model of an HEV along with the supervisory controller are compiled into a real-time interface that enables communication with the emulation layer. On the right side is the emulation layer comprising the real hardware components. Here, the simulated models of the fuel cell and DC/DC converter can be considered as a single unit, and the corresponding hardware component- controllable power source $q 1$ can be used. Similarly, for the battery and supercapacitor, source-sink combination $q 2-s 2$ and source-sink combination $q 3-s 3$ can be used, respectively. Corresponding to the backward simulated part, the power demand or load can be emulated by another source-sink combination $q 4-s 4$. The charging dynamics of the battery and supercapacitor are in accordance with the results given in [16].

For testing the capability of the source-sink combinations in replicating powertrain dynamics, an example for emulating only the backward part of the HEV model can be considered. The simulated behavior and emulated power demand are compared. The performed test results are explained in Figure 14. The source-sink combination $q 4-s 4$ is checked for the emulation of both positive and negative power demand. Here, as demand, the load current from the backward part of the powertrain is used. Its value is positive when the HEV is accelerating or driving at constant velocity and negative when the HEV is braking. During the positive half, the power source $q$ is expected to supply the demand to the power sink s4. A constant current value is set at $q$, and the simulated demand is realized by $s 4$ as motor action. During the negative half, the generator action is realized by $q 4$ as it recuperates energy back to the sink. Here, a constant current is set at the sink.
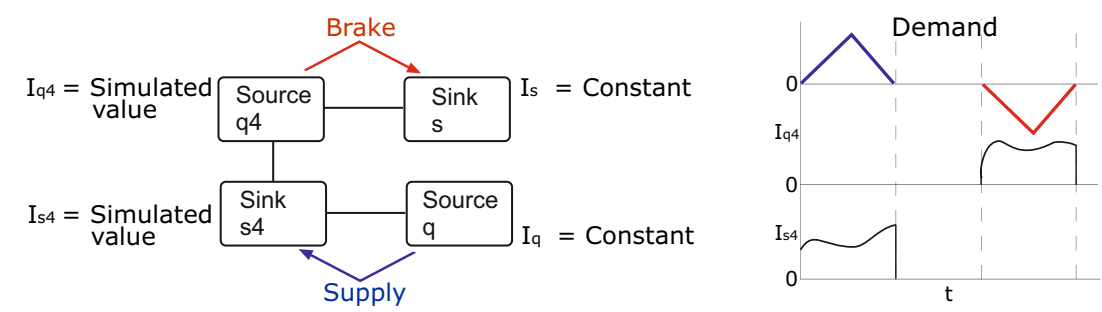

Figure 14. Emulation of motor/generator behavior. 
The result of this test is shown in Figure 15. It can be noted from the figure that the $q 4-s 4$ combination is capable of emulating the motor/generator dynamics. During the positive half of the load cycle, current is drawn by the sink $s_{4}$ (motor mode), and in the negative half of the load cycle, power is supplied by the source $q_{4}$ (generator mode).

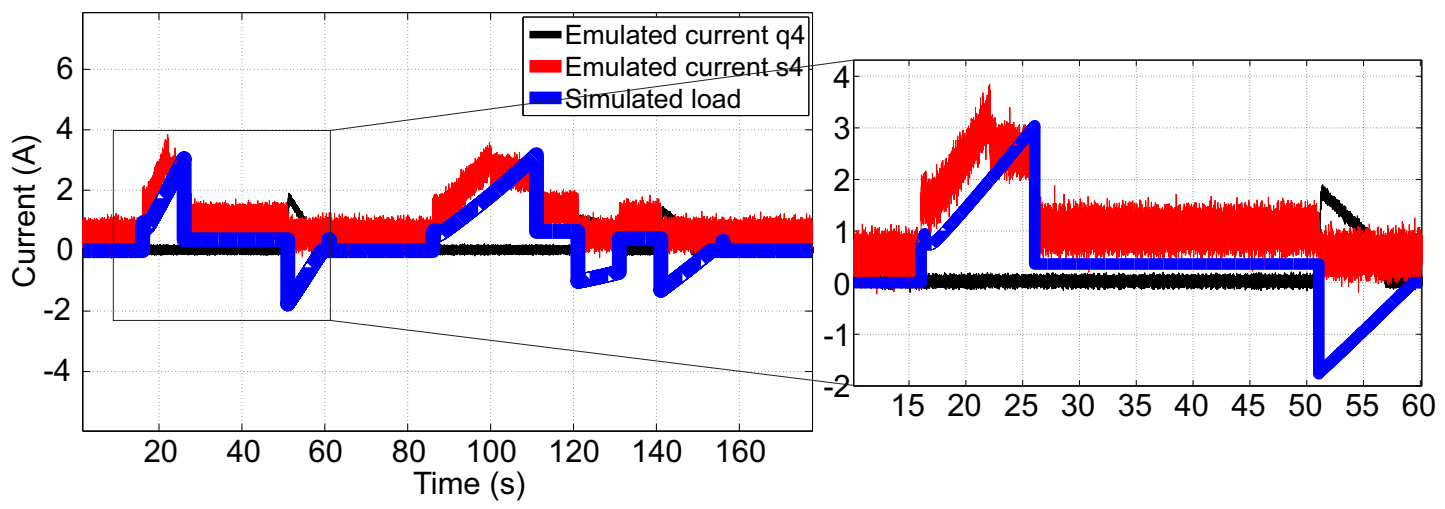

Figure 15. Simulated and experimentally-emulated load current.

This initial test is important to check the ability of power source-sink combinations in emulating not just the motor/generator dynamics, but also the battery and supercapacitor. The constant current supplied by $q$ in the positive half will be replaced by supply from each/either of the sources, as defined by power management. The constant current demanded by the sink in the negative half will be replaced by the demand from each/either of the storage components.

\section{Power Management and Optimization}

As the vehicle considered is a hybrid vehicle and involves multiple sources of power, a supervisory power management control strategy is needed to determine the power flows along single power flow paths to satisfy various objectives while satisfying the power demand at the same time [9].

The developed approach in this work comprises a supervisory controller based on rules designed for a particular drive cycle. The optimization of controller parameters is carried out as a decoupled offline process, the results from which are later embedded online. This power management optimization is implemented on the considered powertrain configuration with three power sources. The developed concept is depicted in Figure 2. The values of battery and supercapacitor SoCs from the forward part of the powertrain, along with the power demand values from the backward part of the powertrain are sent to the mode selection block of the supervisory controller. This block contains IF-THEN rules based on the SoC values and demand. The rule/mode selected corresponds to certain values in the look-up table (LUT) block. These values in the look-up table are used to tune the power management (PM) controller. The controller output is in the form of desired DC/DC converter currents. The LUT block of the supervisory controller contains optimized parameters, as shown in Figure 2. These optimized parameters are generated as a result of a separate offline process, where a multi-objective genetic algorithm-NSGA II is used. The detailed working of each of the supervisory controller blocks [7] is briefly described in the sequel.

The objective of the new developed concept is to tackle multiple facets of HEV control at the same time. A judicious distribution of power between the three sources to ensure proper utilization of each source is considered. This is done by a suitable choice of optimization parameters that ensure that: 
- the required load demand is satisfied at all times,

- the rate of change of the battery and fuel cell current are limited to minimize aging effects,

- the battery current is bound, and the remaining, more dynamic peaks are taken over by the supercapacitor,

- the fuel cell is operated near its optimal range,

- the battery and supercapacitor are never fully charged nor fully discharged,

- the fuel cell delivers maximum power when the SoCs of both the battery and supercapacitor are too low, and

- the bus voltage is held constant to a reference value.

The goal is to integrate optimized parameters that tune the PM controller such that the above criteria are fulfilled. This approach is designed for an assumed drive cycle. If the drive cycle is changed, the designing of modes has to be done accordingly, and new optimal controller parameters will be obtained. The advantage of the developed approach lies in its adaptability. Adaptability here includes the capability to modify/extend it to cases where the drive cycle is not known. The decoupling of the optimization process enables the use of sophisticated algorithms, like multi-objective, global techniques. Only the results are embedded online, thereby reducing the computational effort. The controller takes into account the advantage of the battery-supercapacitor combination and allows the supercapacitor to take over the more dynamic variations in power, thereby extending battery life.

\subsection{Details of the Supervisory Controller}

This section describes the design and working details of the supervisory controller block in Figure 2. In a previous publication [7], the basic working principles of the supervisory controller blocks are explained. A possibility to optimize boundaries and initial conditions is not discussed. In this contribution, optimization of controller parameters along with boundaries and initial conditions is considered. Boundaries refer to the maximum and minimum SoC limits of the battery and supercapacitor, whereas initial conditions refer to the initial SoC levels of the battery and supercapacitor. The role of each parameter in power management and the reasons for optimizing them are also discussed in this contribution. Further details are given in [57]. The hierarchical control concept developed in this work consists of three blocks, as shown in Figure 2: the mode selection block, the look-up table (LUT) block and the power management (PM) controller block. The load is a predefined drive cycle from which three different driving modes can be defined: acceleration and constant velocity (positive load current direction), deceleration (negative load current direction) and standstill (zero load current).

\subsubsection{Mode Selection Block}

The mode selection block takes three inputs from the powertrain model, as shown in Figure 16. The first input is the load current direction $I_{b o a r d}$ for the purpose of distinguishing between the three different driving modes, namely acceleration and constant velocity, deceleration and standstill. The second and third inputs are the battery and supercapacitor SoCs $\left(S_{o} C_{b}\right.$ and $\left.S_{o} C_{s c}\right)$ to decide when the battery/supercapacitor or both the battery and supercapacitor have to be charged/discharged and also when the fuel cell needs to provide maximum power. The output of the mode selection denotes a specific value. For example, when the vehicle is accelerating and both the battery and supercapacitor SoCs have fallen below a certain pre-defined minimum value, the fuel cell is expected to provide the maximum power, so as to satisfy the power demand and also to charge the storage elements. This corresponds to the first case in the mode selection block, and the corresponding value one is sent to the look-up table (LUT) block. When the vehicle velocity is positive, while charging and discharging the battery and supercapacitor, boundary values of maximum and minimum SoC are defined as $B_{\max }, B_{\min }$ and $S C_{\max }, S C_{\min }$. There is a possibility to determine the optimal boundary values, and therefore, these parameters can also be considered for the optimization process. When the vehicle is in deceleration mode and if the fuel cell still continues to provide power, then the excess power is used to charge the 
supercapacitor. The supercapacitor is given a priority here, as according to [58], the dynamics of the powertrain depend mostly on the more dynamic element. In that case, the lower and upper optimal SoC limit of the supercapacitor $\left(S_{o} C_{s c} c_{o p t}\right)$ for the case of deceleration and standstill must be determined. If after mode standstill, the HEV needs to accelerate immediately, then the highest possible reserve should be available from the storage system. If the HEV needs to brake soon after standstill, then the upper limit of the supercapacitor SoC needs to be kept in mind. Similarly, the initial battery and supercapacitor $\mathrm{SoC}$ values can also be considered during the optimization process. The related relations are expressed by parameters according to the algorithms graphically expressed in Figure 16.

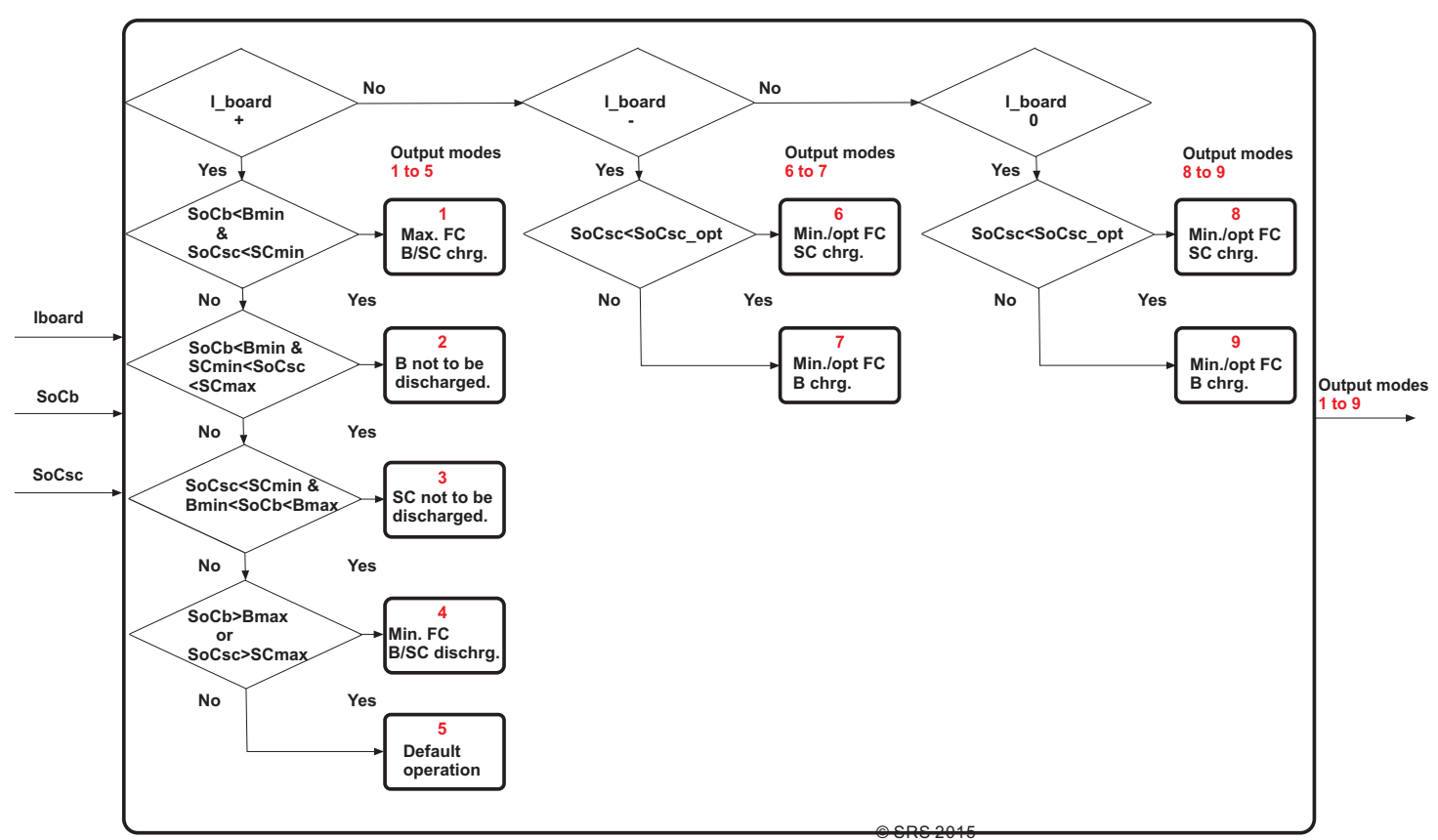

Figure 16. Details of the mode selection block. Fuel cell, battery, and supercapacitor are represented by FC, SC, and B respectively. Charging is defined by chrg. and optimal by opt.

\subsubsection{Look-Up Table Block}

This block consists of four look-up tables for the mode dependent controller parameters: fuel cell current input, power split between the battery and supercapacitor, maximum battery current and minimum battery current. There are LUTs for mode independent parameters, that is boundaries and initial conditions. These parameters can be optimized by specifying the boundaries of variation. The outputs from this block are: the reference fuel cell current depending on its optimal working current value and maximum allowed value given by $I_{F C i n}$; the power sharing between the battery and supercapacitor decided by Powersplit; the battery current, which is limited by $I_{B a t, \text { max }}, I_{B a t, \min }$; and the dynamics of the fuel cell and battery, which are restricted by Ratelimit $_{F C}$, Ratelimit $_{\text {Bat }}$. Thus, the aging problems faced by batteries and fuel cells are minimized by the choice of parameters. These parameters correspond to optimal fuel consumption and are pre-loaded as a result of a separate offline process. As shown in Figure 17, the input to the LUT block from the mode selection block is a number, and it denotes a particular row in each of the four LUTs. For example, when the input is one, the corresponding values of one from all of the LUTs are sent to the PM controller. 


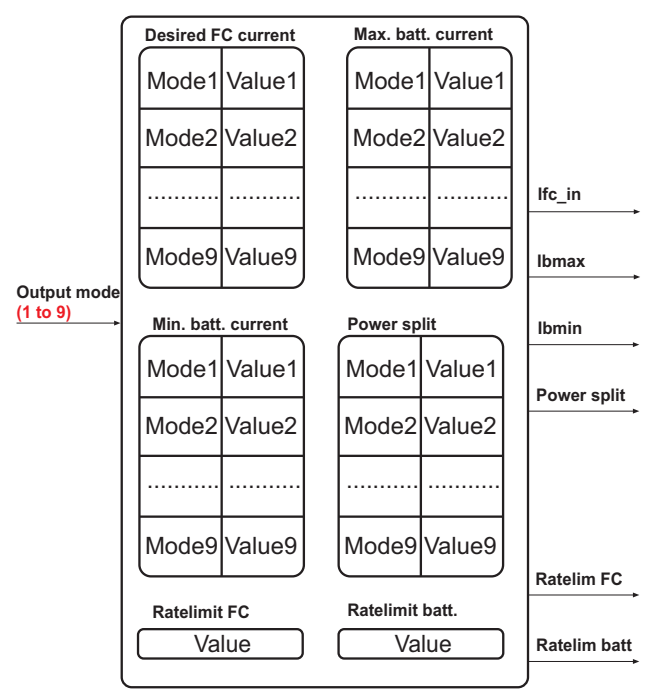

Figure 17. Details of the look-up table block.

\subsubsection{PM Controller Block}

Once the optimal values of $I_{f c i n}$, Powersplit, $I_{B a t, m a x}, I_{B a t, m i n}$, Ratelimit $_{F C}$ and Ratelimit Bat $_{\text {have }}$ been pre-loaded in the LUT block, they can be used for tuning the PM controller online. As detailed in Figure 18, the fuel cell current $I_{f c i n}$ is restricted by a rate limiter before sending it to the DC/DC converter as $I_{f c}$. The rate limiter is used to limit the first derivative (rate) of the signal passing through it, such that the output does not change faster than a specified limit [59]. The load current $I_{b o a r d}$ is divided into fuel cell current $I_{f c}$ and the difference $I_{\text {diff }}$. Here, $I_{\text {diff }}$ is multiplied by the power split value from the LUT. This pre-optimized value of power split influences the charging/discharging dynamics of the battery and supercapacitor. An example of the influence of power split values on the distribution of power between the battery and supercapacitor is given in Figure 19. Here, values between zero and one denote power sharing between the battery and supercapacitor to satisfy the demand. Similarly, values above one and below zero can be used to indicate power flow from the battery to the supercapacitor and vice versa. A suitable choice of power-split values can be made to accommodate all of the possible charging and discharging options of the two storage elements. The actual value of the multiplied variables $I_{\text {diff }} *$ Powersplit is sent to a rate limiter that determines the rising and falling slopes of the current. The output of the rate limiter is sent to a dynamic saturation block that determines the upper and lower limits based on battery minimum and maximum current values $\left(I_{b \min }\right.$ and $\left.I_{b \max }\right)$ from the LUT. Both rate limiter and dynamic saturation are time-dependent components. The output of the saturation block is the battery current $I_{b}$, as shown by the green dotted region in Figure 19, and is sent as the DC/DC converter output. This battery current subtracted from $I_{\text {diff }}$ gives the current that needs to be supplied by the supercapacitor, as shown by the blue dotted region in Figure 19. This is the dynamic part of the current, which needs to be supplied by the supercapacitor, which possesses much higher charging/discharging efficiency than the battery. The outputs from this block, that is the desired currents to be drawn from the three sources, are sent to the DC/DC converters, as shown in Figure 2. The dynamic behavior of the DC/DC converter can be described by:

$$
\mathbf{x}=\left[\begin{array}{l}
I_{L 1} \\
I_{L 2} \\
U_{C l} \\
U_{C 2}
\end{array}\right], \mathbf{u}=\left[\begin{array}{l}
U_{\text {in }} \\
I_{\text {out }}
\end{array}\right], \mathbf{y}=\mathbf{x}
$$


where currents over inductors are denoted by $I_{L 1}$ and $I_{L 2}$ and voltages over capacitances by $U_{C l}$ and $U_{C 2}$. The inputs to the system are the desired current from PM controller $I_{\text {out }}$ and voltage from the fuel cell, battery or supercapacitor $U_{i n}$. The capacitor voltage $U_{C 2}$ denotes the bus voltage, which needs to be held constant, and $I_{L 1}$ the inductor current required to be drawn from the sources. According to [39], the DC/DC converter can be internally controlled by a PI-controller with bus voltage as the reference input, and externally, it can be controlled by the PM controller that sends the desired current output signal to the converter.

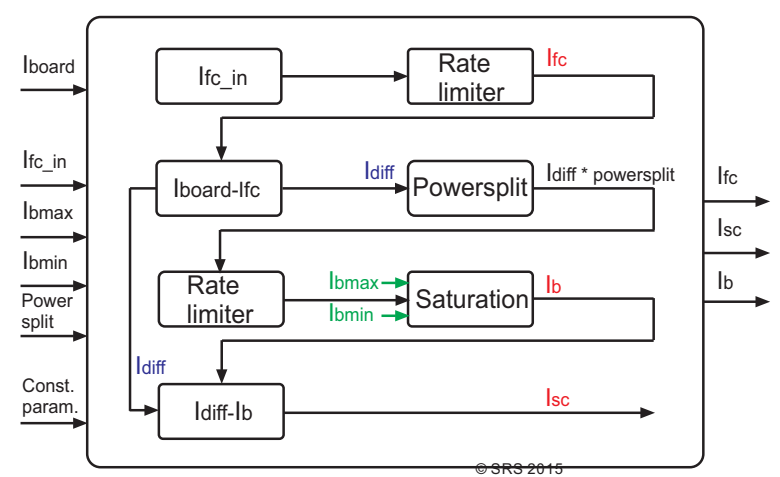

Figure 18. Details of the PM controller block.

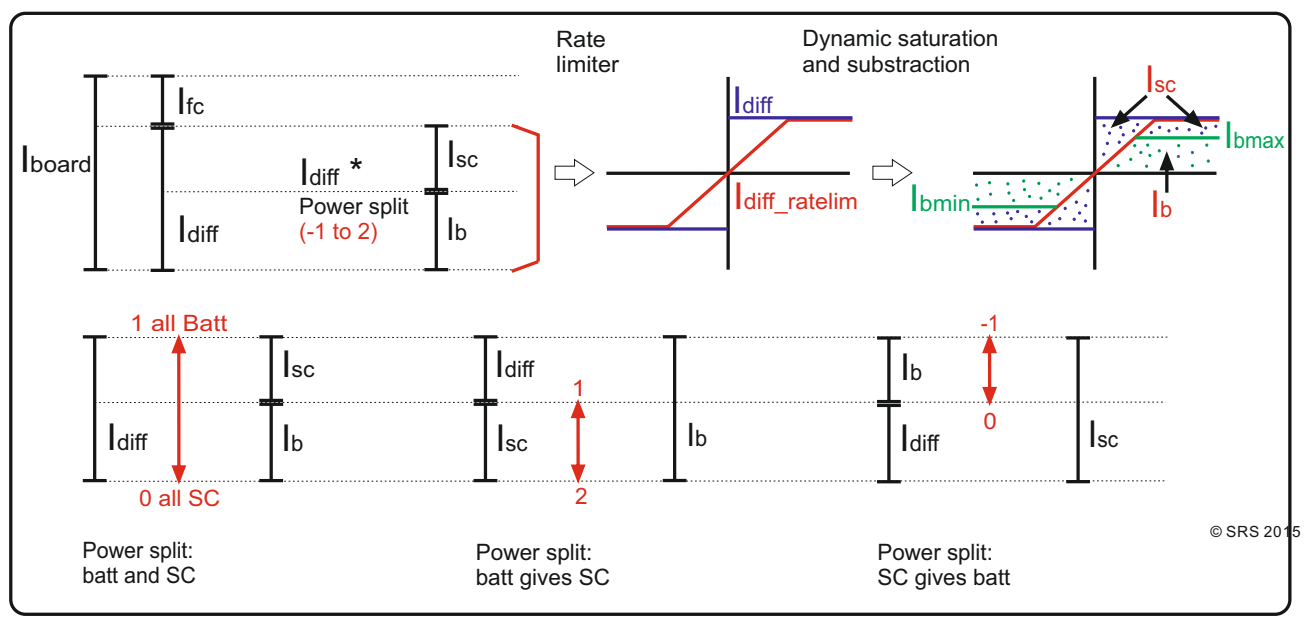

Figure 19. Working of the PM controller block.

\subsection{Optimization as a Decoupled Process}

Optimization problems in hybrid vehicles mainly relate to three types [9]: structural optimization, which finds the best possible powertrain structure; parametric optimization, where the powertrain structure is considered as fixed and the goal is to find the best set of parameters; control system optimization, which finds the best possible supervisory algorithms. This section describes the optimization of the parameters mentioned in the previous section [7]. In this contribution, a parametric optimization is considered for the topology chosen. First, the optimization is carried out at varying supercapacitor sizes for all of the controller parameters along with boundary and initial conditions. Then, a reference size for the supercapacitor is chosen, and the effect of the optimization process is investigated. To sum up, the optimization of the supervisory controller described in the previous section is carried out for two objectives, fuel consumption and SoC deviation, with 46 parameters, four mode dependent for nine modes, 10 mode independent, and the optimization is carried out for four 
different supercapacitor sizes. The optimized results are based on pre-defined drives cycles, here for example, the NEDC drive cycle is used.

In the context of supervisory control strategies, a classification can be made [9], namely heuristic control strategies and optimal control strategies. The advantage of heuristic controllers is their simplicity, but due to the tuning effort required to obtain the optimum fuel economy, optimal control strategies, such as dynamic programming, ECMS, etc., are used. Instead of using an optimal control strategy, which provides a direct optimization of the control input, an offline parametric optimization based on Non-Dominated Sorting Genetic Algorithm (NSGA II) is considered. In [60], NSGA II has been implemented on a hybrid hydraulic powertrain system to generate optimal sizes and control parameters corresponding to given objective functions. As described in [60], the algorithm starts by randomly generating an initial population of possible solutions within the search space, keeping the boundaries of variation of each variable in consideration. The objective functions are evaluated. Each individual is assigned to a rank to generate fronts based on non-dominated sorting. Next, crowding distance is assigned to the individuals to maintain diversity. In the final steps, the selection and assignment of genetic operators, such as crossover and mutation, are performed to obtain the best parameter variables. Details on the adjustment of NSGA II parameter settings, such as crossover probability, mutation rate, etc., can be found in [57]. One of the reasons for selecting this algorithm is its ability to tackle multi-objectives, which might be conflicting in nature [61], which is often the case with hybrid powertrains.

\section{Optimization Goals and Constraints}

The objective function and constraints are given in [9]. The minimization of fuel consumption is given by:

$$
J=\int_{0}^{t_{f}} \dot{m}_{f}(t, u(t)) d t, J \rightarrow \min
$$

where the performance index is denoted by $J$ and fuel mass consumed over a mission of duration $t_{f}$ is denoted by $\dot{m}_{f}$, depending on the system input $u(t)$. The fuel mass consumed $\dot{m}_{f}$ can also be given by the energy required by the fuel cell for the given drive cycle and represented in terms of fuel cell power $P_{F C}$ as follows:

$$
J_{F C}=\int_{t_{0}}^{t_{f}} P_{F C}(t, u(t)) d t, J_{F C} \rightarrow \min .
$$

The other objective is to minimize the SoC deviation, which represents the difference between the initial and final $\mathrm{SoC}$ values of both battery $S o C_{b}$ and supercapacitor $S o C_{s c}$. Generally, the charge sustenance of the storage elements requires small deviations from the nominal value of SoC over the drive cycles. The deviation of the two SoCs, $\Delta S_{o} C_{b}$ and $\Delta S o C_{s c}$, can be considered as an integral constraint [9] by:

$$
J=\phi\left(\Delta\left(S o C_{b}+S o C_{s c}\right)\right)+\int_{0}^{t_{f}} \dot{m}_{f}(t, u(t)) d t
$$

where the penalty term $\phi$ is called the charge-sustaining performance index, given as:

$$
\begin{array}{r}
\phi\left(\Delta S o C_{b}\right)=\alpha\left|\left(S_{S o} C_{b}\left(t_{0}\right)-S o C_{b}\left(t_{f}\right)\right)\right| \\
\phi\left(\Delta S o C_{s c}\right)=\beta\left|\left(S o C_{s c}\left(t_{0}\right)-S o C_{s c}\left(t_{f}\right)\right)\right|
\end{array}
$$

with $\Delta S o C_{s c}$ and $\Delta S o C_{b}$ as the SoC deviations of the supercapacitor and battery at time interval $t_{0}$ to $t_{f}$.

\section{Simulated and Emulated Results}

In this subsection the effect of the developed power management control concept is discussed. From Figure 20a, the basic working principles of the power management strategy become clear using an NEDC drive cycle as the example. The battery current is limited, and the more dynamic fluctuations 
are taken over by the supercapacitor. The fuel cell is operated close to its efficient operating point as calculated in [28]. To evaluate the feasibility of the emulation hardware in realizing the dynamics of the powertrain, the simulated results are compared to emulation results. In Figure 20b, the charging of the battery and supercapacitor is shown. This is in accordance with the simulated battery and supercapacitor current, as shown in Figure 20a. The curves in Figure 20b demonstrate an inversion of the values shown in Figure 20a. The negative parts of load represent charging or an increase in sink $s 2$ and $s 3$ currents and positive parts, discharging or an increase in source $q 2$ and $q 3$ currents. Here, only the emulation of charging current is shown. Similarly, the emulation of supply and regeneration dynamics using $s$ and $q$ is explained with Figure 15.

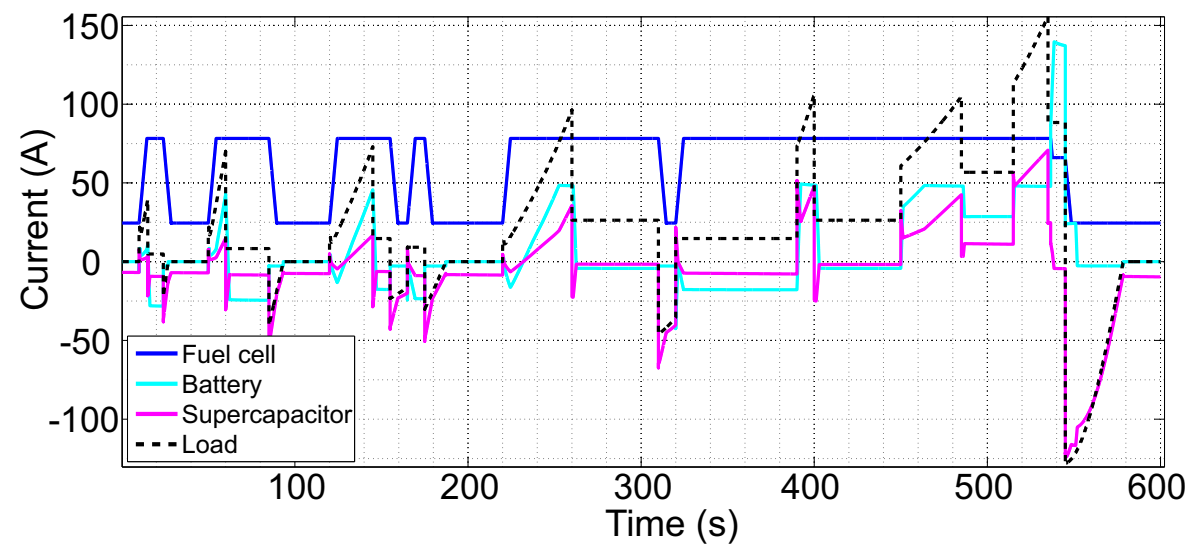

(a)

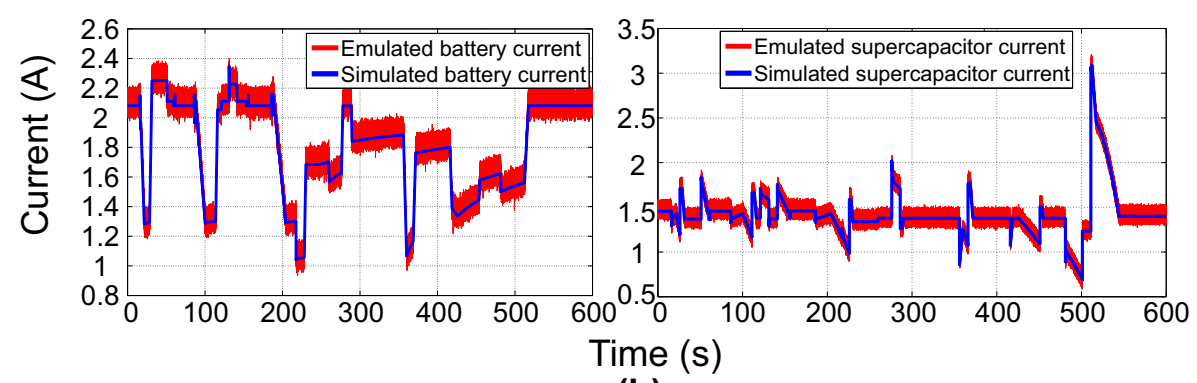

(b)

Figure 20. (a) Simulated source and load current; (b) experimentally-emulated battery and supercapacitor current.

Here, the emulation of both positive and negative parts of the load demand by $q 4-s 4$ is given. During the positive half of the load cycle, current is drawn by the sink $s 4$ (motor mode), and in the negative half of the load cycle, power is supplied by the source $q 4$ (generator mode). Therefore, the emulated $q 4$ current, which is an absolute value, can be seen as an inversion of simulated value. From Figures 15 and 20, a good coincidence between model behavior and emulation can be noted. Thus, the dynamics of the simulated models of components together with the supervisory controller can be realized using the emulated experimental setup. Corresponding to the three sources, the DC/DC converter current outputs as defined by the power management controller are sent to $q 1, q 2-s 2$ and $q 3-s 3$. The simulated load current from the backward modeled part is sent to $q 4-s 4$.

Next, the effect of the optimized controller parameters on the HEV dynamics is analyzed. For the two chosen objectives, namely minimization of fuel consumption and SoC deviation of both the battery and supercapacitor, the results are analyzed in the following manner:

- To investigate the influence of component sizing, the variation of the two objective functions for different supercapacitor sizes is shown in Figure 21. 
- A supercapacitor size is chosen, and the convergence of the two conflicting objectives for the chosen supercapacitor is shown in Figure 22.

- The preference between the two objectives is varied, and its influence on SoC variation is analyzed.
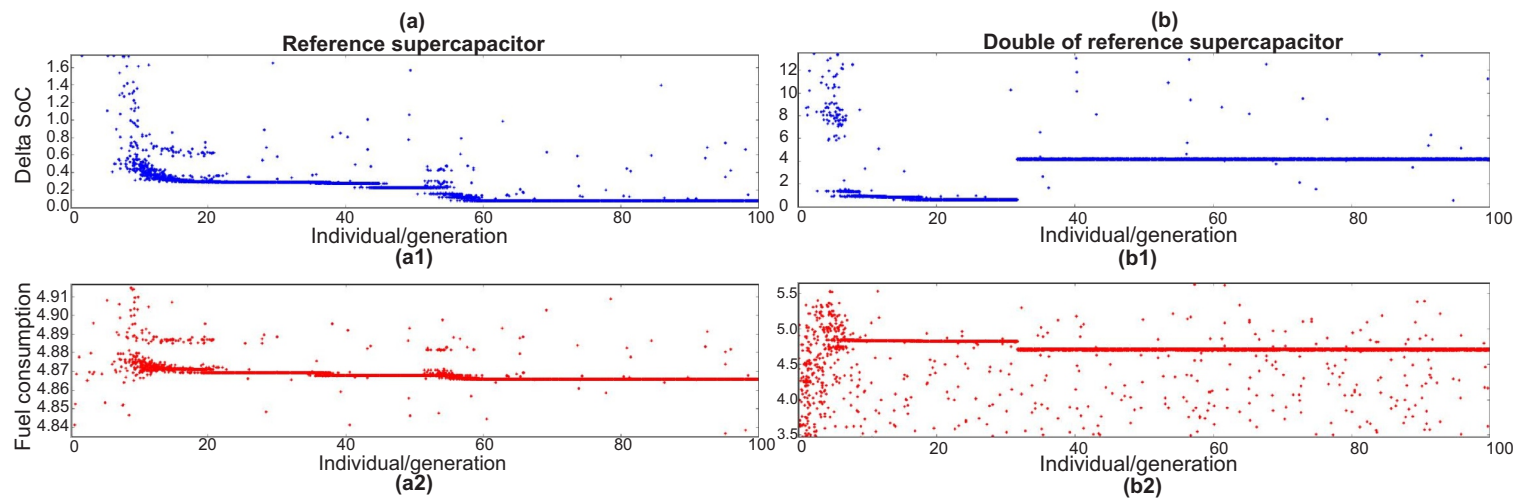

(c)
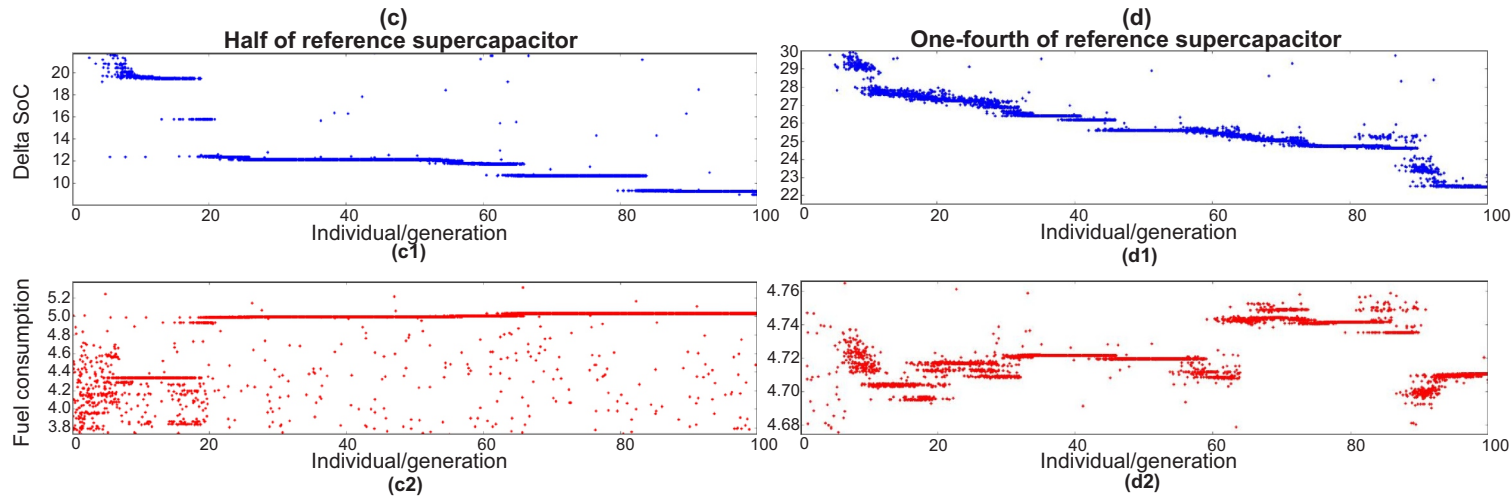

Figure 21. Objective function convergence for (a) the reference supercapacitor, (b) double the reference supercapacitor, (c) half the reference supercapacitor and (d) one-fourth the reference supercapacitor.

To demonstrate (and to learn about) the principal behaviors, four supercapacitor sizes are chosen for comparison: the reference, double the reference, half the reference and one-fourth the reference. The optimization runs for all of the supercapacitor sizes can be seen in Figure 21. As shown in Figure 21b, with the double-sized supercapacitor, a slight improvement in fuel consumption values is noted (Figure 21b2) at the cost of the deterioration of $\Delta S o C$ values (Figure 21b1). However, this does not provide an optimal solution for the total objective function, and further iteration steps of the optimization algorithm are required. As shown in Figure 21c, with the half-sized supercapacitor, a prominent improvement in $\triangle S o C$ is noted (Figure 21c1). The fuel consumption is not minimized within the shown iteration steps. Finally, in Figure 21d, with one-fourth the supercapacitor, dynamic and fluctuating behavior is noted in Figure 21d1,d2. Within the shown iteration steps, minimization, particularly of fuel consumption values, is not possible. Thus, with such small supercapacitor sizes, the control task is difficult, and the overall system might become unstable.

In Figure 22, conflicting solutions for the two objective functions for the reference supercapacitor can be seen. This results from the principle contradictions in the task of fuel consumption minimization and SoC sustenance. However, a convergence of the total objective function can be obtained. From Figure 22, it can be noted that although minimum values for $\triangle S o C$ are obtained, the values chosen for fuel consumption are not necessarily the minimum values. Lower fuel consumption values that did not satisfy the minimum $\triangle S o C$ are rejected, so a compromising solution for both objectives can be obtained. 


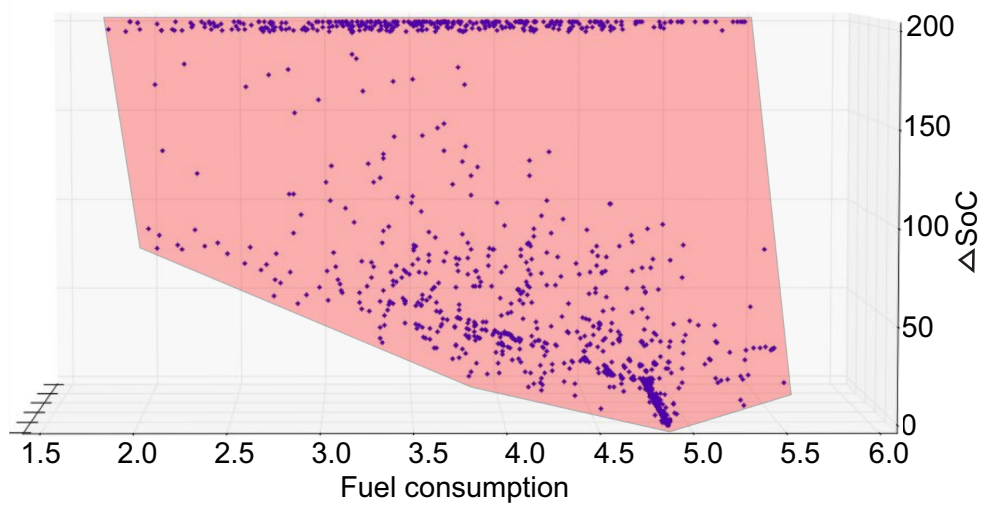

Figure 22. Conflict of objectives.

In the next step, the parameters corresponding to the optimized values obtained from NSGA II are integrated in the online power management control strategy. Three supercapacitor sizes, double (denoted in green), half (denoted in blue) and one-fourth (denoted in black), are compared to the reference size (denoted in red) to analyze the influence on $\mathrm{SoC}$ and on fuel consumption. In Figure 23, the SoCs of the battery and supercapacitor are shown along with the corresponding fuel cell output power and distinguished for small and large supercapacitors.
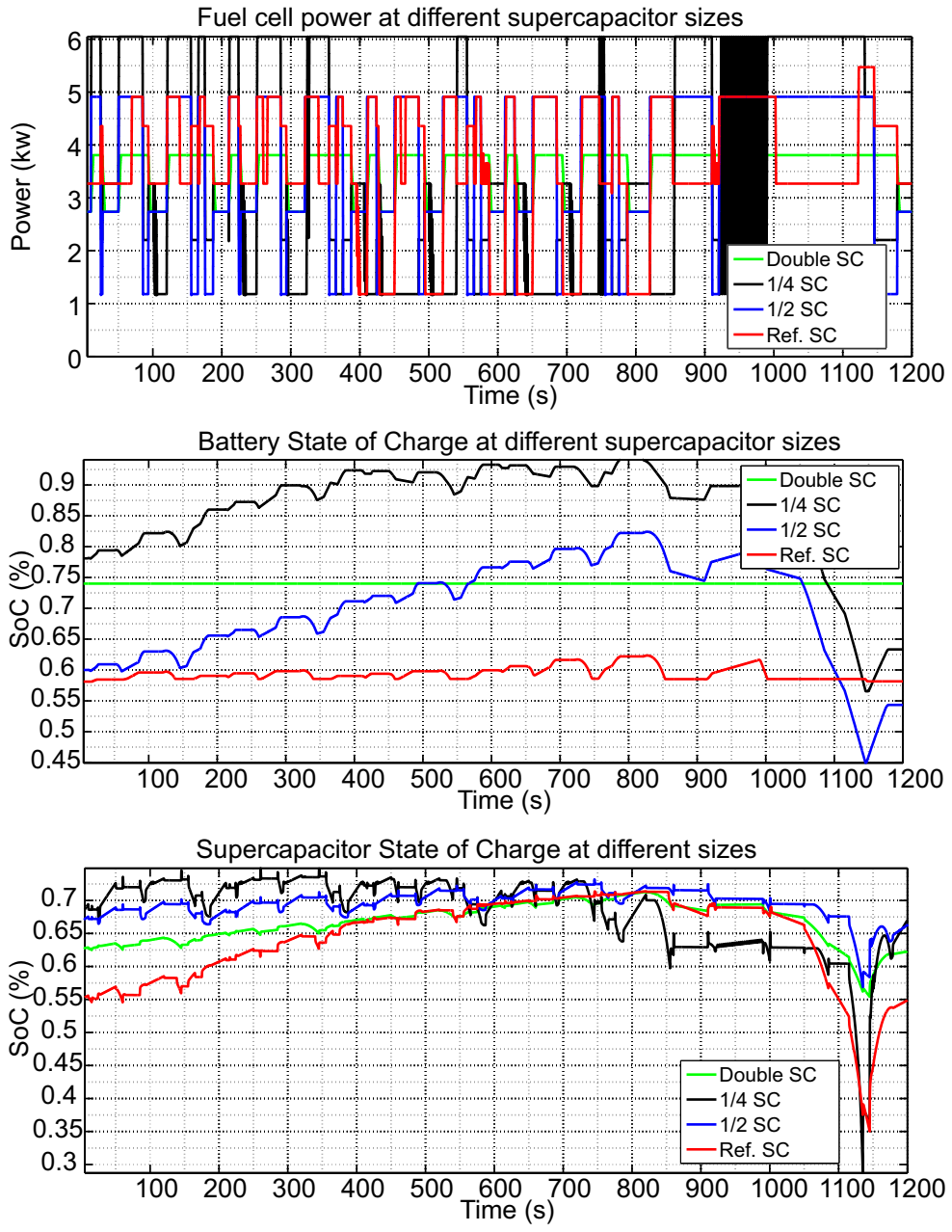

Figure 23. Effect of optimization parameters at different supercapacitor sizes (simulated). 
Smaller supercapacitors: From the battery SoC, it can be seen that the battery is gradually charged in the beginning, so as to gain reserves for following the high demanding part of the drive cycle, due to the insufficient storage capacity of the supercapacitor. Then, the battery is discharged till its lowest SoC value. From the supercapacitor SoC, it can be seen that smaller sizes cause more fluctuations. With the one-fourth size, the response is very dynamic. From the fuel cell output power, it can be seen that power supplied by the fuel cell is more for smaller supercapacitors than larger ones. Transient behavior is noted with the smallest supercapacitor.

Larger supercapacitors: When the supercapacitor is doubled, the battery SoC is constant, meaning that the battery is not required. In this case, the large supercapacitor has sufficient storage capacity. The supercapacitor SoC curve is flatter and less fluctuating in comparison with smaller supercapacitor SoCs. From the fuel cell output power, it becomes clear that the power supplied by fuel cell is also least here.

Thus, with the double-sized supercapacitor, the most desirable performance can be achieved, whereas by using a one-fourth-sized supercapacitor, undesirable effects may result. These undesirable effects need to be avoided, keeping the size and cost of the powertrain in mind. The reference size can be considered as a suitable option.

Next, the priority between the two objectives is varied for the reference supercapacitor. For the realization of different requirements, the priorities are assigned as given in the following three cases: Case 1: priority distribution decided by NSGA II (denoted in green); Case 2: high priority on fuel consumption and less priority on $\triangle S o C$ (denoted in blue); Case 3: high priority on fuel consumption with least priority on $\triangle S O C$ (denoted in red). These three cases can be obtained by analyzing the effects of parameter changes during different stages of optimization. In the first case, the parameters correspond to those obtained at the end of optimization. In the second and third cases, the parameters correspond to those obtained in the intermediate stages. In Figure 24, the SoC variations of the battery and supercapacitor can be seen along with the corresponding fuel cell power.

Case 1 is the standard case and can be used as a reference for the comparison of Cases 2 and 3. Case 2 (blue curve): From the battery SoC, it can be seen that the battery SoC is mostly sustained and discharged only towards the end. The supercapacitor is charged from the fuel cell. By comparing battery $\triangle S o C$ to Case 1 (green curve), it is observed that $\Delta S o C$ in Case 1 is lower than in Case 2. This is because in Case 2, the optimization objective-fuel economy is taken into account, but the second objective-battery $\triangle S o C$ is sacrificed.

Case 3 (red curve): From the battery SoC, it can be seen that the battery is more depleted than in Cases 1 and 2. The supercapacitor is charged from the battery. By comparing battery $\triangle S o C$ with Cases 1 and 2, it is observed that $\triangle S o C$ in both Cases 1 and 2 is lower than in Case 3. 

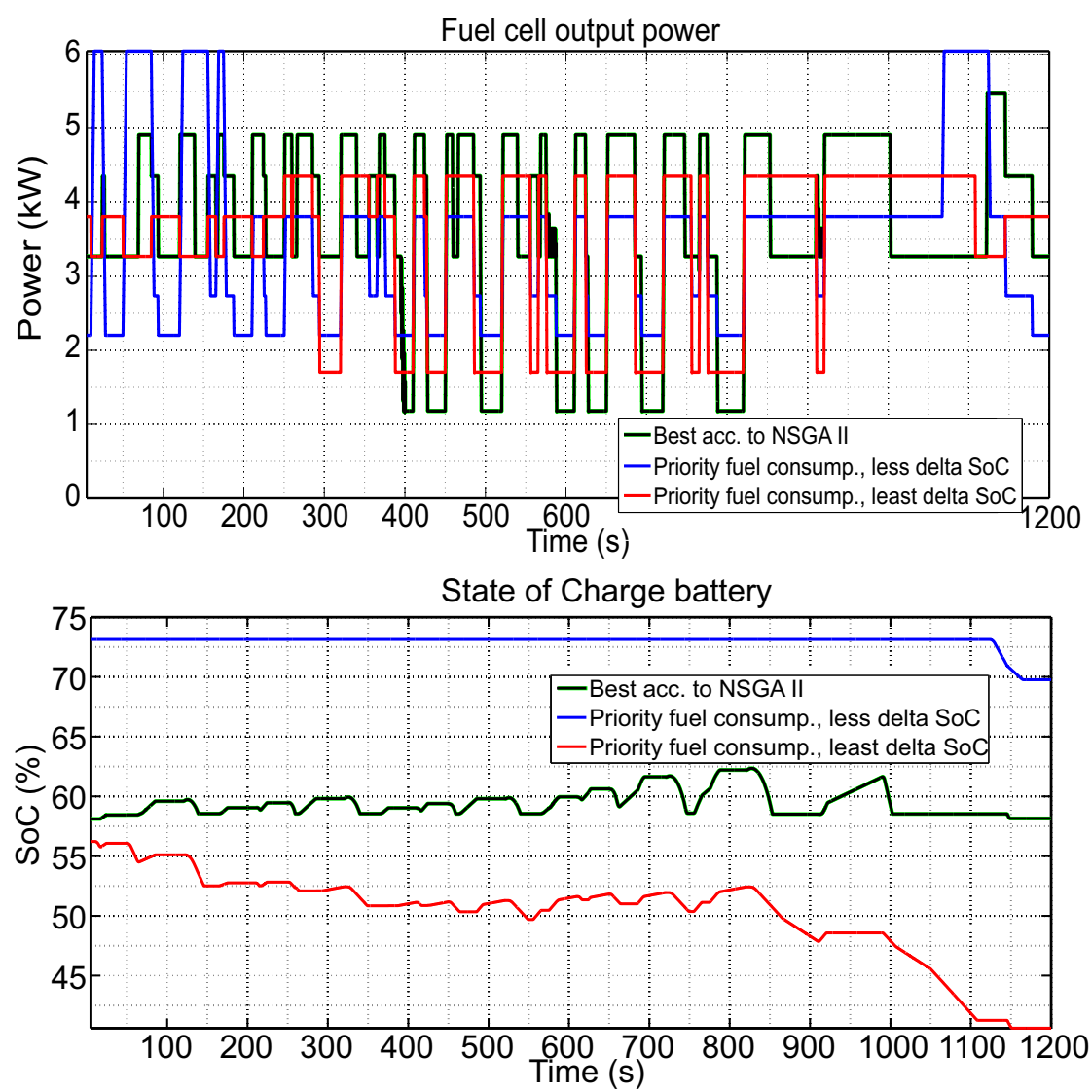

State of Charge supercapacitor

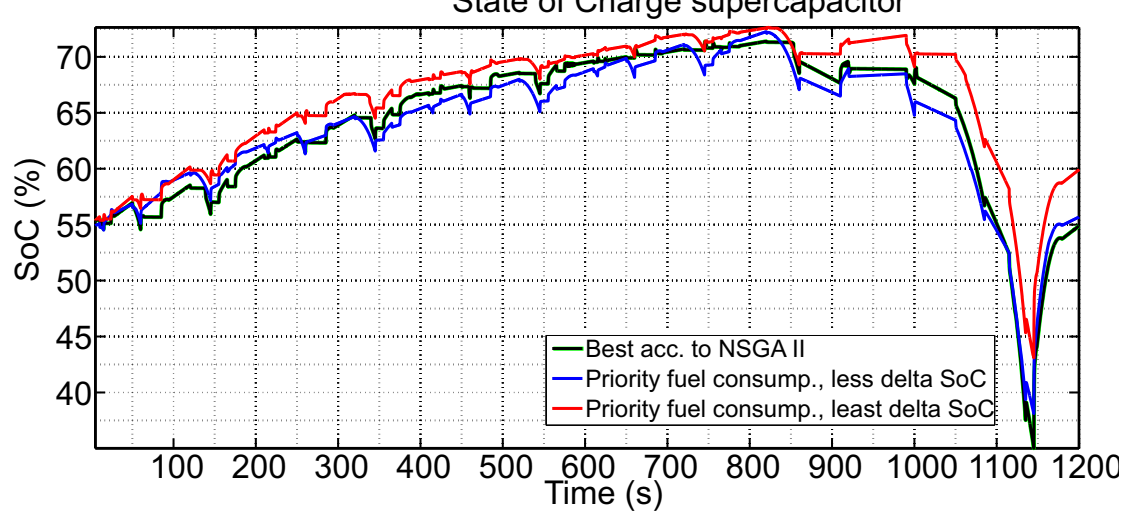

Figure 24. Effect of varying priorities between optimization goals (simulated).

Thus, when priority is assigned in the order fuel consumption followed by supercapacitor $\triangle S o C$ followed by battery $\triangle S o C$, the battery is more often discharged, as shown in the above results. By changing the priority between the objectives, further possibilities can be investigated. However, as the objectives are conflicting in nature, a compromise has to be made.

In Figure 25, the total energy consumption corresponding to non-optimal power management, optimal power management and optimal power management as a function of different supercapacitor sizes is shown. Total energy consumption denotes the energy of the fuel cell plus the energy of the battery and supercapacitor, which can be added or subtracted from the total energy depending on the charge/discharge. When the supercapacitor size is increased, energy consumption will be reduced. This results directly from the ability of exchanging dynamic load peaks. With smaller supercapacitors, the energy consumption is distinctively higher. 


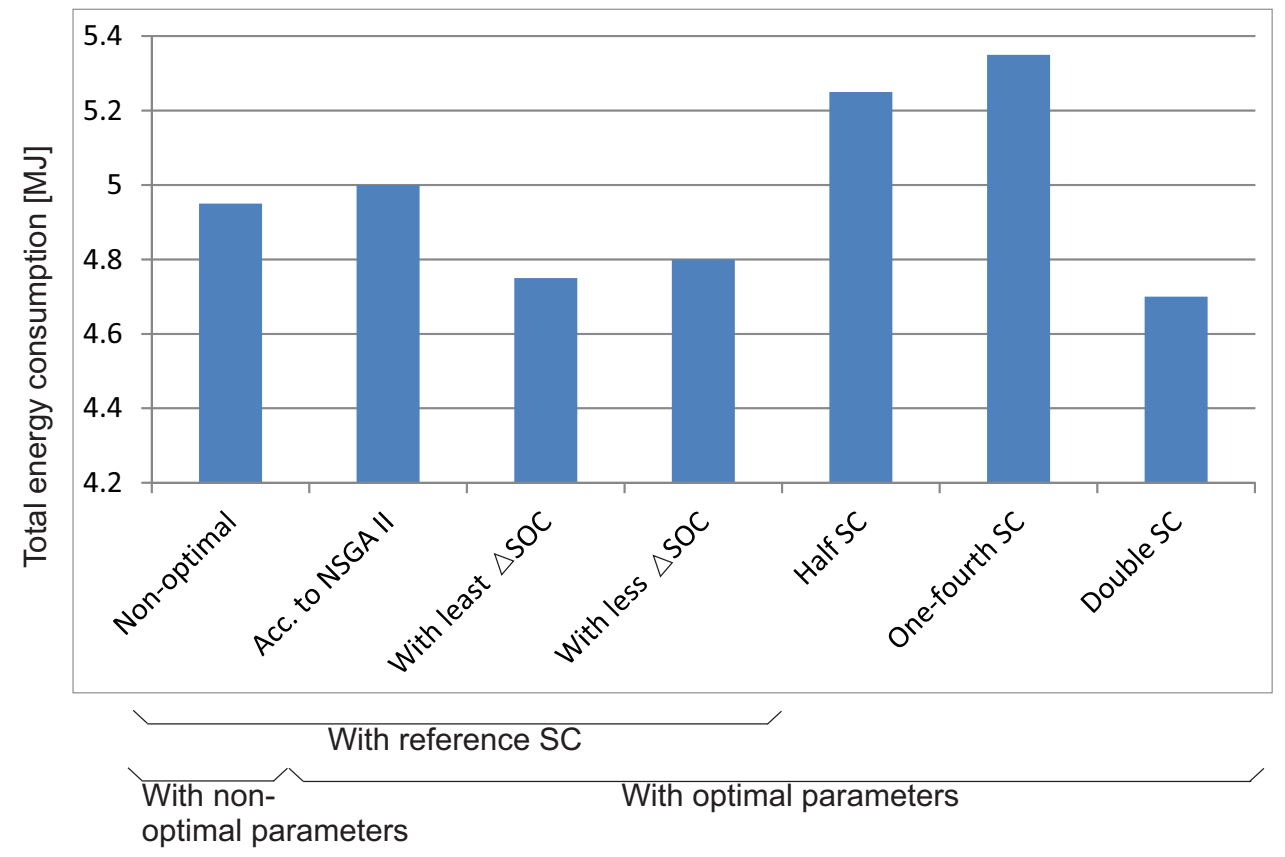

Figure 25. Comparison of all results in terms of energy consumption.

\section{Summary and Conclusions}

In this contribution, first, a brief literature review is presented. This emphasizes the role of rule-based power management and optimization strategies. The existing strategies lack the ability to provide optimal solutions in terms of multiple objectives online. Along with online optimization, the adaption of optimized control rules to real driving data is an issue. To overcome the deficits of existing strategies, namely the integration of multiple objectives along with adaptability to real driving patterns, an optimized power management controller for a three-source HEV is developed and realized experimentally by an emulation test-rig. The newly-introduced power management concept is capable of determining the optimal power distribution between the three sources online, such that the dynamic part of the load is supplied by the supercapacitor and battery current is limited. The decoupling of the optimization process from the online part enables the use of offline-implementable, multi-objective algorithms. From the simulation results, it can be concluded that: multiple, even conflicting optimization objectives can be integrated in this control strategy; by changing the priority between the objectives, further options can be investigated; and by a suitable selection of parameters, all three sources can be operated within desired working ranges while satisfying the load demand at the same time. Due to the modular structure of the power management optimization concept, an extension of the concept can be easily realized by integrating more LUTs with sets of optimized parameters. Real (unknown dynamic) drive cycles can be used to generate these parameters offline to be embedded online.

Acknowledgments: This contribution is an outcome of the research activities at the Chair of Dynamics and Control (SRS), University of Duisburg-Essen. The authors would like to thank the scientific and technical staff of the chair who have supported the work, particularly with the HiL test-rig. The authors would also like to thank Timo Rauhut whose master thesis was based on the concepts detailed in this contribution.

Author Contributions: Both authors contributed equally to the paper, whereby the corresponding author was mainly responsible for the initial writing, figures and literature research and the second author for structuring and organizing, reviewing and summarizing the entire contribution and research.

Conflicts of Interest: The authors declare no conflict of interest. 


\section{References}

1. Khaligh, A.; Li, Z. Battery, ultracapacitor, fuel cell, and hybrid energy storage systems for electric, hybrid electric, fuel cell, and plug-in hybrid electric vehicles: State of the art. IEEE Trans. Veh. Technol. 2010, 59, 2806-2814.

2. Aharon, I.; Kuperman, A. Topological overview of powertrains for battery-powered vehicles with range extenders. IEEE Trans. Power Electron. 2012, 26, 868-876.

3. Paladini, V.; Donateo, T.; Risi, A.; Laforgia, D. Super-capacitors fuel-cell hybrid electric vehicle optimization and control strategy development. Energy Convers. Manag. 2007, 48, 3001-3008.

4. Khoucha, F.; Benbouzid, M.; Kheloui, A. An optimal fuzzy logic power sharing strategy for parallel hybrid electric vehicles. In Proceedings of the IEEE Vehicle Power and Propulsion Conference, Lille, France, 1-3 September 2010.

5. Li, Q.; Chen, W.; Li, Y.; Liu, S.; Huang, J. Energy management strategy for fuel cell/battery/ultracapacitor hybrid vehicle based on fuzzy logic. J. Electr. Power Energy Syst. 2012, 43, 514-525.

6. Moulik, B.; Söffker, D. Optimal rule-based powermanagement for online, real-time applications with multiple sources and objectives: A review. Energ. Open Access Energy Res. Eng. Policy J. 2015, 8, 9049-9063.

7. Moulik, B.; Söffker, D. Online powermanagement with embedded optimization for a multi-source hybrid with dynamic power sharing between components. In Proceedings of the ASME Dynamic Systems and Control (DSC) Conference, Columbus, OH, USA, 28-30 October 2015.

8. Bayindir, K.; Gozukucuk, M.; Teke, A. A comprehensive overview of hybrid electric vehicle: Powertrain configurations, powertrain control techniques and electronic control units. Energy Convers. Manag. 2010, 52, 1305-1313.

9. Guzzella, L.; Sciarretta, A. Vehicle Propulsion Systems: Introduction to Modeling And Optimization, 2nd ed.; Springer: Berlin/Heidelberg, Germany, 2007.

10. Lin, C.; Filipi, Z.; Louca, L.; Peng, H.; Assanis, D.; Stein, J. Modeling and control of a medium-duty hybrid electric truck. Int. J. Veh. Des. 2004, 11, 349-370.

11. Meintz, A.; Ferdowsi, M. Control strategy optimization for a parallel hybrid electric vehicle. In Proceedings of the IEEE Vehicle Power and Propulsion Conference (VPPC), Harbin, China, 3-5 September 2008.

12. Sorrentino, M.; Rizzo, G.; Arsie, I. Analysis of a rule-based control strategy for on-board energy management of series hybrid vehicles. Control Eng. Pract. 2011, 19, 1433-1441.

13. Hofman, T.; Steinbuch, M. Rule-based energy management strategies for hybrid vehicles. Int. J. Electr. Hybrid Veh. 2007, 1, 71-94.

14. Zhang, B.; Chen, Z.; Mi, C.; Murphey, Y. Multi-objective parameter optimization of a series hybrid electric vehicle using evolutionary algorithms. In Proceedings of the IEEE Vehicle Power and Propulsion Conference (VPPC), Dearborn, MI, USA, 1-3 September 2010.

15. Serrao, L.; Onori, S.; Sciarretta, A.; Guezennec, Y.; Rizzoni, G. Optimal energy management of hybrid electric vehicles including battery aging. In Proceedings of the American Control Conference (ACC), San Francisco, CA, USA, 29 June-1 July 2011.

16. Marx, M. Multiobjective Obtimization of the Power Flow Control of Hybrid Electric Power Train Systems within Simulation and Experimental Emulation Applications. Ph.D. Thesis, University of Duisburg-Essen, Essen, Germany, 2014.

17. Mihael, C.; Pavković, D.; Petrić, J. A Control-oriented simulation model of a power-split hybrid electric vehicle. Appl. Energy 2013, 101, 121-133.

18. Torres, J.; Gonzalez, R.; Gimenez, A.; Lopez, J. Energy management strategy for plug-in hybrid electric vehicles, a comparative study. Appl. Energy 2014, 114, 816-824.

19. Fotouhi, A.; Yusof, R.; Rahmani, R.; Mekhilef, S.; Shateri, N. A review on the applications of driving data and traffic information for vehicles' energy conservation. Renew. Sustain. Energy 2014, 37, 822-833.

20. Lin, C.; Jeon, S.; Peng, H.; Moo Lee, J. Driving pattern recognition for control of hybrid electric trucks. Veh. Syst. Dyn. 2004, 42, 41-58.

21. Lukic, S.; Cao, J.; Bansal, R.; Rodriguez, F.; Emadi, A. Energy storage systems for automotive applications. IEEE Trans. Ind. Electron. 2008, 55, 2258-2267. 
22. Lukic, S.; Wirasingha, S.; Rodriguez, F.; Cao, J.; Emadi, A. Power management of an ultracapacitor/battery hybrid energy storage system in an HEV. In Proceedings of the IEEE Vehicle Power and Propulsion Conference (VPPC), Windsor, ON, Canada, 6-8 September 2006.

23. Baisden, A.; Emadi, A. ADVISOR-based model of a battery and an ultra-capacitor energy source for hybrid electric vehicles. IEEE Trans. Veh. Technol. 2004, 53, 199-205.

24. Cao, J.; Emadi, A. A new battery/ultracapacitor hybrid energy storage system for electric, hybrid, and plug-in hybrid electric vehicles. IEEE Trans. Power Electron. 2012, 27, 122-132.

25. Hu, X.; Murgovski, N.; Johannesson, L.; Egardt, B. Comparison of three electrochemical energy buffers applied to a hybrid bus powertrain with simultaneous optimal sizing and energy management. IEEE Trans. Transp. Syst. 2014, 15, 1193-1205.

26. Valera, J.; Iglesias, I.; Peña, A.; Martin, A.; Sánchez, J. Integrated modeling approach for highly electrified HEV. Virtual design and simulation methodology for advanced powertrain prototyping. In Proceedings of the International Battery, Hybrid and Fuel Cell Electric Vehicle Symposium (EVS 24), Stavanger, Norway, 13-16 May 2009.

27. Debert, M.; Colin, G.; Chamaillard, Y.; Guzzella, L.; Ketfi-Cherif, A.; Bellicaud, B. Predictive energy management for hybrid electric vehicles-Prediction horizon and battery capacity sensitivity. In Proceedings of the IFAC Symposium Advances in Automotive Control, Munich, Germany, 11 July 2010.

28. Özbek, M.; Wang, S.; Marx, M.; Söffker, D. Modeling and control of a PEM fuel cell system: A practical study based on experimental defined component behavior. J. Process Control 2013, 23, 282-293.

29. Eker, J.; Hagander, P.; Årzén, K. A feedback scheduler for real-time controller tasks. Control Eng. Pract. 2000, $12,1369-1378$.

30. Hofmann, M.; Eckardt, B.; März, M.; Frey, L. Effizienzoptimierung integrierter elektrischer Antriebssysteme fuer Hybrid- und Elektrofahrzeuge. In Proceedings of the Elektromobilausstellung (EMA)—Fachtagung-Wettbewerbe, Aschaffenburg, Germany, 8-9 September 2010.

31. Oei, D.; Adams, J.; Kinnelly, A. Direct-Hydrogen-Fueled Proton-Exchange-Membrane Fuel Cell System for Transportation Applications; No. DOE/CE/50389-503; Ford Motor Co.: Dearborn, MI, USA, 1997.

32. He, H.; Xiong, R.; Guo, H.; Li, S. Comparison study on the battery models used for the energy management of batteries in electric vehicles. Energy Convers. Manag. 2012, 64, 113-121.

33. Waag, W.; Käbitz, S.; Sauer, D. Experimental investigation of the lithium-ion battery impedance characteristic at various conditions and aging states and its influence on the application. Appl. Energy 2014, 102, 885-897.

34. Knauff, M.C.; Dafis, C.J.; Niebur, D.; Kwatny, H.G.; Nwankpa, C.O. Simulink model for hybrid power system test-bed. In Proceedings of the IEEE Electric Ship Technologies Symposium, Arlington, VA, USA, 21-23 May 2007.

35. Chen, M.; Rincon-Mora, G. Accurate electrical battery model capable of predicting runtime and I-V performance. IEEE Trans. Energy Convers. 2006, 21, 504-511.

36. Rahimi-Eichi, H.; Chow, M.Y. Adaptive parameter identification and state-of-charge estimation of lithium-ion batteries. In Proceedings of the IECON 2012-38th Annual Conference on IEEE Industrial Electronics Society, Montreal, QC, Canada, 25-28 October 2012.

37. Fărcaş, C.; Petreuş, D.; Ciocan, I.; Palaghită, N. Modeling and simulation of supercapacitors. In Proceedings of the 15th International Symposium for Design and Technology of Electronics Packages, Gyula, Hungary, 17-20 September 2009.

38. Johansson, P.; Andersson, B. Comparison of Simulation Programs for Supercapacitor Modelling. Master's Thesis, Chalmers University of Technology, Gothenburg, Sweden, 2008.

39. Uzunoglu, M.; Alam, M.S. Dynamic modeling, design and simulation of a PEM fuel cell/ultra-capacitor hybrid system for vehicular applications. Energy Convers. Manag. 2007, 48, 1544-1553.

40. Brusa. Brusa Elektronik AG. Available online: http://www.brusa.eu/ (accessed on 11 October 2015).

41. Veneri, O.; Capasso, C.; Patalano, S. Experimental study on the performance of a ZEBRA battery based propulsion system for urban commercial vehicles. Appl. Energy 2016, doi:10.1016/j.apenergy.2016.01.124 (In press).

42. Kim, T.; Qiao, A. A hybrid battery model capable of capturing dynamic circuit characteristics and nonlinear capacity effects. IEEE Trans. Energy Convers. 2011, 26, 1172-1180.

43. Liu, K.; Zhu, C.; Lu, R.; Chan, C. Improved study of temperature dependence equivalent circuit model for supercapacitors. IEEE Trans. Plasma Sci. 2013, 41, 1267-1271. 
44. Emadi, A. Handbook of Automotive Power Electronics and Motor Drives; CRC Press: Boca Raton, FL, USA, 2005.

45. Thacker, B.; Doebling, S.; Hemez, F.; Anderson, M.; Pepin, J.; Rodriguez, E. Concepts of Model Verification and Validation; No. LA-14167; Los Alamos National Lab: Los Alamos, NM, USA, 2004.

46. Capasso, C.; Veneri, O. Experimental analysis on the performance of lithium based batteries for road full electric and hybrid vehicles. Appl. Energy 2014, 136, 921-930.

47. Chou, P.; Park, C.; Park, J.; Pham, K.; Liu, J. B\#: A battery emulator and power profiling instrument. In Proceedings of the International Symposium on Low Power Electronics and Design, Seoul, Korea, 25-27 August 2003.

48. Gao, F.; Blunier, B.; Bouquain, D.; Miraoui, A. Model based DC power source emulator for electrical and hybrid electrical vehicles drive train tests. In Proceedings of the IEEE Vehicle Power and Propulsion Conference (VPPC), Chicago, IL, USA, 6-9 September 2011.

49. Ferreira, A.; Pomilio, J.; Spiazzi, G.; de Araujo Silva, L. Energy management fuzzy logic supervisory for electric vehicle power supplies system. IEEE Trans. Power Electron. 2008, 23, 107-115.

50. Parker-Allotey, N.; Bryant, A.; Palmer, P. The application of fuel cell emulation in the design of an electric vehicle powertrain. In Proceedings of the Power Electronics Specialists Conference, Recife, Brazil, 16 June 2005.

51. Gao, F.; Blunier, B.; Simoes, M.; Miraoui, A.; El-Moudni, A. PEM fuel cell stack hardware-in-the-loop emulation using DC/DC converter design. In Proceedings of the Electrical Power and Energy Conference (EPEC), Montreal, QC, Canada, 22-23 October 2009.

52. Marsala, G.; Pucci, M.; Vitale, G.; Cirrincione, M.; Miraoui, A. A prototype of a fuel cell PEM emulator based on a buck converter. Appl. Energy 2009, 86, 2192-2203.

53. Höcherl and Hackl. Battery Emulation with NL Source-Sink, H \& H Customer Application 12. Available online: http:/ / www.hoecherl-hackl.com/customer-applications.html (accessed on 25 November 2015).

54. Rao, S.; Chandorkar, M. Electrical load emulation using power electronic converters. In Proceedings of the TENCON 2008-2008 IEEE Region 10 Conference, Hyderabad, India, 19-21 November 2008.

55. Marx, M.; Özbek, M.; Söffker, D. Power management of a hybrid electric powertrain system-Design, power flow control and optimization targets. Int. J. Powertrains 2014, 3, 221-241.

56. Liu, Y.; Marx, M.; Moulik, B.; Söffker, D. Experiment-based simulation and optimization of wind powertrain systems based on electric power flow emulation. In Proceedings of the First Conference for Wind Power Drives, Aachen, Germany, 19-20 March 2013.

57. Rauhut, T. Power Management and Optimization of a Multi-Source Hybrid Powertrain for Real Time Applications (Power-Management und Optimierung eines Multi-Quelle-Hybrid-Antriebes für Echtzeitanwendungen). Master's Thesis, University of Duisburg-Essen, Essen, Gemany, 2015.

58. Karbaschian, M.; Söffker, D. Review and comparison of power management approaches for hybrid vehicles with focus on hydraulic drives. Energies 2014, 7, 3512-3536.

59. MathWorks. Rate Limiter. Available online: http://www.mathworks.com (accessed on 26 January 2016).

60. Moulik, B.; Karbaschian, M.; Söffker, D. Size and parameter adjustment of a hybrid hydraulic powertrain using a global multi-objective optimization algorithm. In Proceedings of the IEEE Vehicle Power and Propulsion Conference (VPPC), Beijing, China, 15-18 October 2013.

61. Deb, K.; Jain, P.; Gupta, N.K.; Maji, H.K. Multiobjective placement of electronic components using evolutionary algorithms. IEEE Trans. Compon. Packag. Technol. 2004, 27, 480-492.

(C) 2016 by the authors; licensee MDPI, Basel, Switzerland. This article is an open access article distributed under the terms and conditions of the Creative Commons Attribution (CC-BY) license (http://creativecommons.org/licenses/by/4.0/). 